\title{
Dynamics of a close-loop controlled MEMS resonator
}

\author{
Abdulrahman Seleim • Shahrzad Towfighian • \\ Emmanuel Delande • Eihab Abdel-Rahman • \\ Glenn Heppler
}

Received: 16 July 2011 / Accepted: 23 November 2011

(C) Springer Science+Business Media B.V. 2011

\begin{abstract}
The dynamics of a close-loop electrostatic MEMS resonator, proposed as a platform for ultra sensitive mass sensors, is investigated. The parameter space of the resonator actuation voltage is investigated to determine the optimal operating regions. Bifurcation diagrams of the resonator response are obtained at five different actuation voltage levels. The resonator exhibits bi-stability with two coexisting stable equilibrium points located inside a lower and an upper potential wells. Steady-state chaotic attractors develop in-
\end{abstract}

\author{
A. Seleim $(\bowtie)$ \\ Industrial and Manufacturing Systems Engineering, \\ University of Windsor, Windsor, Ontario, Canada \\ e-mail: seleim@uwindsor.ca \\ S. Towfighian \\ Mechanical and Industrial Engineering, University \\ of Toronto, Toronto, Ontario, Canada \\ e-mail: sherryt@mie.utoronto.ca \\ E. Delande \\ LAGIS (CNRS FRE 3303), Ecole Centrale de Lille, \\ Villeneuve d'Ascq, France \\ e-mail: emmanuel.delande@centraliens-lille.org \\ E. Abdel-Rahman · G. Heppler \\ Systems Design Engineering, University of Waterloo, \\ Waterloo, Ontario, Canada \\ E. Abdel-Rahman \\ e-mail: eihab@uwaterloo.ca \\ G. Heppler \\ e-mail: heppler@uwaterloo.ca
}

side each of the potential wells and around both wells. The optimal region in the parameter space for mass sensing purposes is determined. In that region, steadystate chaotic attractors develop and spend most of the time in the safe lower well while occasionally visiting the upper well. The robustness of the chaotic attractors in that region is demonstrated by studying their basins of attraction. Further, regions of large dynamic amplification are also identified in the parameter space. In these regions, the resonator can be used as an efficient long-stroke actuator.

Keywords Chaos · Bifurcation - MEMS .

Electrostatic actuators

\section{Introduction}

MEMS are used widely as sensors due to their small size, high sensitivity and precision, and low cost. Mass sensing is an important commercial application of MEMS sensors. One approach to realize MEMS mass sensors is to place a selective recipient material on the surface of a micro-beam. When the target analyte adheres to the recipient surface, it changes the mass of the beam. The change in the mass is then determined by either measuring the change in static position of the beam [1], or tracking a change in dynamic behavior as a result of that change in mass. The change in dynamic behavior can be a shift in the natural frequency $[2,3]$, 
the location of a bifurcation point $[4,5]$, or the eigenmode of an array of beams [6].

Yin and Epureanu [7] were able to measure experimentally small variations in the mass of a cantilever beam by detecting changes in the shape of its chaotic attractor. Chaos in dynamic systems was first observed in the late nineteenth century by Poincaré; however, useful applications of this interesting phenomenon did not emerge until the nineties [8]. The metrics of chaotic attractors are highly sensitive to variations in the system parameters [9] which makes them an ideal platform for parameter identification. Epureanu el al. [10] were able to detect damage in thermo-shielding panels undergoing chaotic oscillations. Ghafari et al. [11] used the Lyapunov exponent of the chaotic oscillations of rolling element bearings to detect bearing faults. Wu et al. [12] used the 3-dimensional fractal dimension of the fetal cortical surface to assess the level of cortical development and to detect morphologic abnormalities in fetuses. Combining the enhanced sensitivity of the chaotic attractor metrics and MEMS sensors can take micro-mass sensing to a new dimension.

Chaos in MEMS was first observed by Bienstman et al. [13] in an electrostatically actuated impact resonator. They used a simple fixed-fixed beam driven by a voltage larger than the pull-in voltage. When the beam pulls in, a short circuit is introduced. As a result, the voltage drops and the mechanical stiffness becomes larger than the electrostatic force, thus forcing the beam away from the electrode. When contact is lost, the electric force starts building up and the beam pulls in again. This switching-like operation produces large periodic motions. Chaos was observed in the system, and thus tracked to be avoided. Wang et al. [14] realized a MEMS resonator excited by two non-overlapping comb-drives, thus creating a bi-stable Duffing oscillator. They measured chaotic oscillations experimentally around a drive frequency of $6 \mathrm{kHz}$, which is the natural frequency of the system. However, there were large mismatches between the model and experiment. The mismatch was mainly due to their inability to estimate the system parameters. DeMartini et al. [15] used a similar structure and were also able to produce chaotic motions experimentally around $20 \mathrm{kHz}$, which is about 1.15 times the natural frequency of the system.

De and Aluru [16] reported chaos in a model of an open-loop electrostatically actuated parallel-plate resonator prior to pull-in. Najar et al. [17] reported an incomplete cascade of period-doubling bifurcations in a similar model just before pull-in. They showed that chaos cannot occur in open-loop electrostatic actuators due to the occurrence of a homoclinic bifurcation which leads to rapid dynamic pull-in.

Liu et al. [18] observed period-doubling bifurcations and chaos in a model of a close-loop controlled micro-resonator. The close-loop system was designed and realized to serve as a magnetic probe tip actuator for a probe-based micro-disc drive [19]. Towfighian et al. [20] studied this system and found an asymmetric two-well potential with two distinct chaotic attractors; one of which occurs predominantly in the lower-well, and a second that visits a lower-well orbit and a twowell orbit.

Electrostatically-actuated micro-beams are gaining widespread use as actuators, resonators, and sensors. The requirements for each of these uses are disparate and in some cases contradictory. For example, micropositioning requires repeatability of motion along a trajectory, while sensing requires high sensitivity; relatively large responses to relatively small stimuli. A major obstacle in the use of the electrostatically actuated micro-beams is the presence of pull-in instability which takes place when the electrostatic force overcomes the elastic restoring force in the beam. Recently, closed-loop control was used to retard or eliminate the pull-in instability $[19,21]$.

In particular, Towfighian et al. [21] demonstrated experimentally a closed loop controlled electrostatic micro-actuator with an operating range over $90 \%$ of the gap. This actuator can realize large-orbit oscillations within the electrostatic field. Since electrostatic forces are highly nonlinear, the ramifications of these large motions are not obvious and require further investigation.

In this paper, we undertake a qualitative study of the Towfighian et al. [20, 21] actuator that maps the actuation parameter-space to classify available motion patterns, identify phenomena arising due to large motions within strongly non-linear electrostatic fields, and to delineate regions where it can be used as a micropositioner or periodic resonator and regions where it can be deployed as a chaotic resonator to serve as a platform for a high-sensitivity mass sensor. Toward that end, bifurcation diagrams are obtained for a range of excitation voltage amplitudes and frequencies. Lyapunov exponents are found to verify the existence of chaotic attractors and the basins of attraction of those 
attractors are also found to determine the safe basin of motions.

\section{The close-loop electrostatic resonator}

The motions of a micro-cantilever beam electrostatic resonator, Fig. 1 can be described using a single-mode approximation. The single degree-of-freedom equation of motion can be written as [20-22]:

$\ddot{d}=-\frac{k}{m} d+\frac{\beta V^{2}}{m(g-d)^{2}}$

where $d, k, m, \beta, V$, and $g$ are the displacement of the beam tip, the effective stiffness of the beam, the effective mass of the beam, the electro-mechanical coupling coefficient, the voltage drop across the capacitor gap, and the gap distance, respectively. The electrostatic force term in (1) is highly non-linear. In fact, the electrostatic force grows and approaches infinity as the resonator displacement grows and approaches the size of the capacitor gap $d \rightarrow g$. On the other hand, the beam stiffness $k$ is finite, which causes the static equilibrium of the resonator to lose stability at about one-third of the gap $g$ in a phenomenon known as

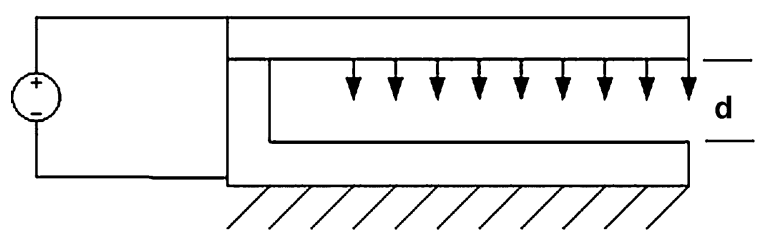

Fig. 1 Electrostatically actuated cantilever beam static pull-in. The static and dynamic response of uncontrolled electrostatic MEMS resonators have been studied extensively by many researchers, for example Najar et al. in [17, 23].

With the introduction of close-loop control [20, 21], stable beam motions can be extended to over $90 \%$ of the gap. Further, the close loop actuator can be configured to exhibit two stable equilibria instead of the single equilibrium available under open loop conditions. Large motions and bi-stability fundamentally change the dynamics of MEMS electrostatic resonators, thereby suggesting a re-examination of the types of behavior available in the parameter space and their potential applications.

Figure 2 shows a block diagram of the close-loop electrostatic resonator under study [21]. The plant is a MEMS microcantilever beam with length, width, and thickness $L, w$, and $h$, respectively. The plant input is the actuation voltage $V_{a}$. A vibrometer (Polytech MSV 400) is used to measure the velocity of the beam tip. The velocity of the tip is the plant output that is used in feedback path to generate the control voltage $V_{s}$ and close the loop. The controller is implemented using analog electronics. The close-loop system equations are [20]:

$$
\begin{aligned}
\dot{d}= & v \\
\dot{v}= & -\mu v \\
& +\frac{-c_{3} d-c_{4} d^{2}-c_{5} d^{3}+c_{6} G^{2}\left(V_{a} \sqrt{\alpha}-V_{s}\right)^{2}}{c_{0}+c_{1} d+d^{2}} \\
& \dot{V}_{s}=-r\left(V_{s}-\frac{d}{1-d} \Psi \sqrt{\alpha}\right)
\end{aligned}
$$

Fig. 2 Block diagram of the close-loop system

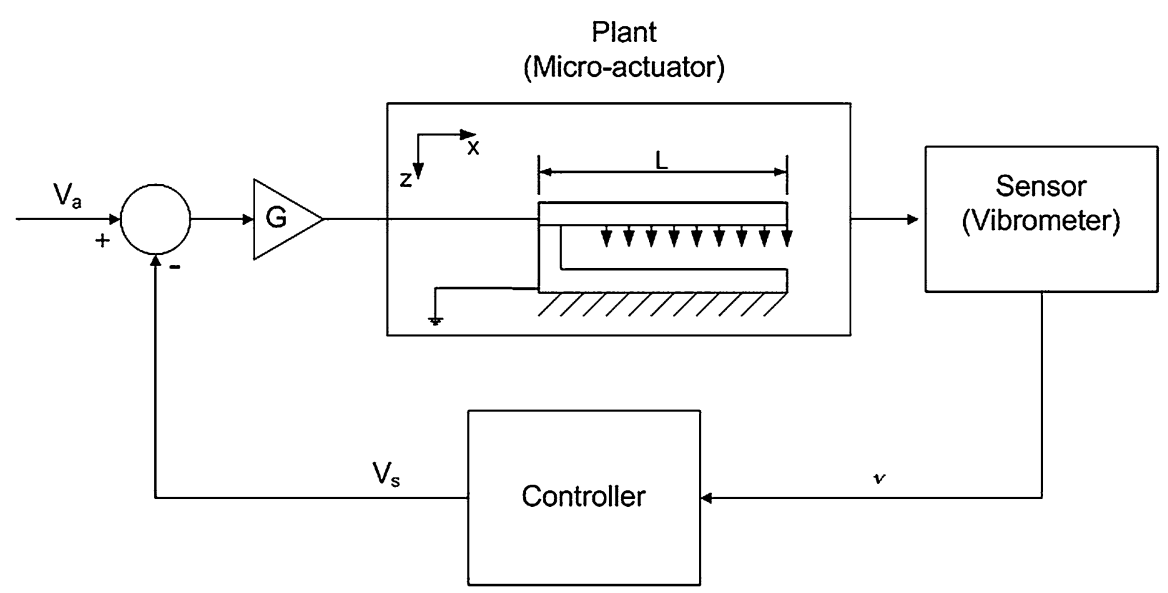


A. Seleim et al.

Table 1 System parameters used in simulations

\begin{tabular}{ll}
\hline Parameter & Value \\
\hline$L$ & $200 \mu \mathrm{m}$ \\
$w$ & $80 \mu \mathrm{m}$ \\
$h$ & $4.5 \mu \mathrm{m}$ \\
$g$ & $3 \mu \mathrm{m}$ \\
$\mu$ & 0.73 \\
$r$ & 100 \\
$G$ & 0.8 \\
$\Psi$ & $3 \mathrm{~V}$ \\
$\rho$ & $2331 \mathrm{~kg} / \mathrm{m}^{3}$ \\
$E$ & $166 \mathrm{GP}$ \\
$\alpha$ & $0.0002 \mathrm{~V}^{-2}$ \\
\hline
\end{tabular}

where $d(t)$ and $v(t)$ represent the displacement and velocity of the tip of the beam in the $z$ direction, Fig. 2, $V_{s}(t)$ is the non-dimensional control voltage, $V_{a}(t)=$ $V_{\mathrm{DC}}+V_{\mathrm{AC}} \sin (\Omega t)$ is the actuation voltage, $c_{i}$ are nondimensional modal coefficients obtained from a onemode Galerkin expansion, $\mu$ is the non-dimensional damping coefficient, $r$ is the controller damping, $G$ is a voltage gain, $\Psi$ is a displacement gain, and $\alpha$ is the electromechanical coupling coefficient. The tip position, voltage, and time are non-dimensionalized with respect to the capacitor gap $g$, the electromechanical coupling coefficient $\alpha$, and the time constant of the beam $T=\sqrt{\frac{\rho A L^{4}}{E I}}$ respectively, where $\alpha$ has the dimensions of volts ${ }^{-2}, A=h w, E$ is Young's modulus, and $I=\frac{w h^{3}}{12}$. The first two equations govern the position and velocity of the tip of the beam, and the third governs the controller voltage. The system parameters are listed in Table 1.

Towfighian et al. [20] used these system parameters to find a region of bi-stability in the range $V_{\mathrm{DC}}=$ $[108,113] \mathrm{V}$. At $V_{\mathrm{DC}}=110 \mathrm{~V}$, the controlled microbeam has five static equilibrium positions. These positions were determined by setting the time derivatives in (2)-(4) equal to zero and solving the resulting algebraic system. The stability of each equilibrium point was then determined by calculating the eigenvalues of the Jacobian matrix of the system evaluated at the given fixed position. Out of the five equilibrium positions, only four are physical. The fifth equilibrium position is physical because it indicates a tip position larger than 1 beyond the bottom electrode located at $d=1$. For the other four equilibrium positions, two were found to be stable foci, while the other two were
Table 2 Stable positions and corresponding natural frequency

\begin{tabular}{|c|c|c|c|}
\hline$V_{\mathrm{AC}}$ & Equilibrium position & Natural frequency $\omega_{i}$ & Stability \\
\hline \multirow[t]{4}{*}{$2 \mathrm{~V}$} & 0.371037 & 1.9742 & Stable \\
\hline & 0.681634 & & Unstable \\
\hline & 0.863958 & 4.43694 & Stable \\
\hline & 0.984587 & & Unstable \\
\hline \multirow[t]{4}{*}{$2.5 \mathrm{~V}$} & 0.371144 & 1.97343 & Stable \\
\hline & 0.681441 & & Unstable \\
\hline & 0.864018 & 4.44096 & Stable \\
\hline & 0.984588 & & Unstable \\
\hline \multirow[t]{4}{*}{$3 \mathrm{~V}$} & 0.371276 & 1.97250 & Stable \\
\hline & 0.681206 & & Unstable \\
\hline & 0.864092 & 4.44586 & Stable \\
\hline & 0.984588 & & Unstable \\
\hline \multirow[t]{4}{*}{$3.5 \mathrm{~V}$} & 0.371432 & 1.97139 & Stable \\
\hline & 0.680928 & & Unstable \\
\hline & 0.864092 & 4.44586 & Stable \\
\hline & 0.984589 & & Unstable \\
\hline \multirow[t]{4}{*}{$4 \mathrm{~V}$} & 0.371612 & 1.9701 & Stable \\
\hline & 0.680607 & & Unstable \\
\hline & 0.86428 & 4.45832 & Stable \\
\hline & 0.98459 & & Unstable \\
\hline
\end{tabular}

found to be unstable saddles. Each of the two stable equilibrium positions has its own natural frequency which is dependent on the input DC voltage.

Bi-stability introduces rich dynamics to the system response and increases the likelihood of chaos. In the presence of a combined DC and AC input voltage, the equilibrium positions and natural frequencies are determined by the RMS value of the total actuation voltage. The natural frequency of oscillations around a given equilibrium position is equal to the imaginary component of the complex pair of the eigenvalues of the Jacobian evaluated at that position. Table 2 shows the equilibrium positions and the corresponding natural frequencies for $V_{\mathrm{DC}}=110 \mathrm{~V}$ and $V_{\mathrm{AC}}=[2,2.5,3,3.5,4] \mathrm{V}$.

\section{Analysis procedure}

To investigate available dynamic regimes within the parameter space, bifurcation diagrams are obtained at five values of the excitation voltage amplitudes $V_{\mathrm{AC}}$ 
with the frequency of excitation $\Omega$ acting as the control parameter. The bifurcation diagrams were generated using a two-sided Poincaré section at the plane $v=0$. The value of $d$ at the intersection points with this section represent the maximum and minimum positions of the beam tip, therefore allowing us to describe the relative location of the orbit as well as its size. The procedure used to construct the bifurcation diagrams is presented in Appendix A.

The Lyapunov exponents were calculated to verify the existence of the chaotic attractors and to characterize them. The Lyapunov exponent is a measure of expansion or contraction of perturbations around a given trajectory. A dissipative system becomes chaotic when at least one Lyapunov exponent is positive [9] due to the presence of an exponentially growing process inside the contracting space.

The non-autonomous system equations (2)-(4) were transformed to an autonomous system by adding a state variable $\theta$ and an equation to describe its evolution over time:

$\dot{d}=v$

$\dot{v}=-\mu v+$

$\frac{-c_{3} d-c_{4} d^{2}-c_{5} d^{3}+c_{6} G^{2}\left(\sqrt{\alpha}\left(V_{\mathrm{DC}}+V_{\mathrm{AC}} \cos \theta\right)-V_{s}\right)^{2}}{c_{0}+c_{1} d+d^{2}}$

$\dot{V}_{s}=-r\left(V_{s}-\frac{d}{1-d} \Psi \sqrt{\alpha}\right)$

$\dot{\theta}=\Omega$

The Lyapunov exponents were then calculated following the algorithm used in [9]. The complete procedure for calculating the exponents is described in Appendix B. The Lyapunov exponents were calculated for large branches of chaotic attractors. The value of the maximum Lyapunov exponent was used to verify the existence of chaos and to measure the relative activity of the chaotic attractor.

\section{Results}

\subsection{System response at $V_{\mathrm{AC}}=2 \mathrm{~V}$}

Figure 3 shows the bifurcation diagram for an $\mathrm{AC}$ amplitude of $V_{\mathrm{AC}}=2 \mathrm{~V}$ where solid lines represent stable orbits and dotted lines represent unstable orbits.

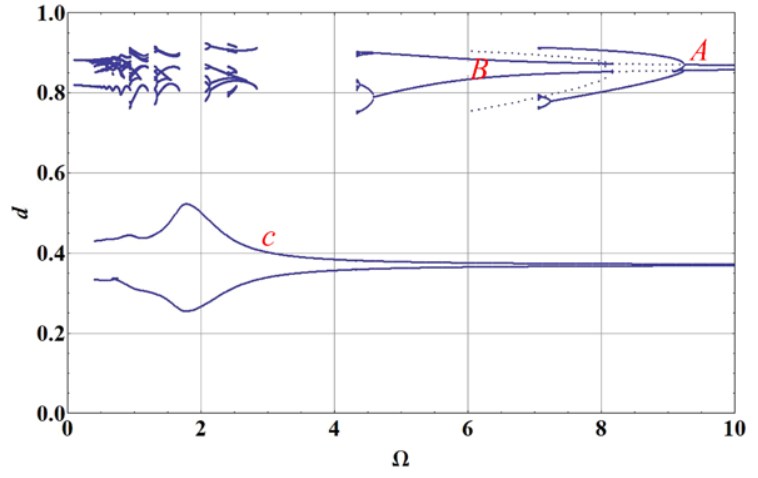

Fig. 3 The bifurcation diagram for $V_{\mathrm{AC}}=2 \mathrm{~V}$

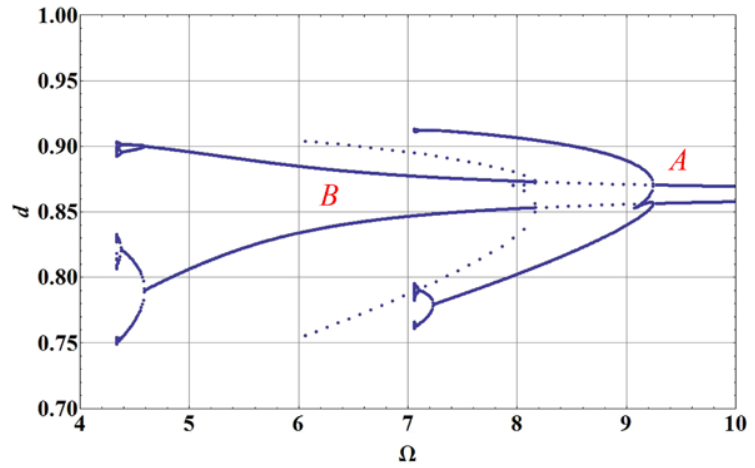

Fig. 4 The bifurcation diagram for the upper-well orbits at $V_{\mathrm{AC}}=2 \mathrm{~V}$

The presence of a two-well potential field is obvious in the appearance of attractors limited to the lowerwell and others limited to the upper-well. No two-well orbits were observed at this excitation level. The effect of the asymmetry in the two potential wells can be seen obvious in the relative size and location along the frequency spectrum of those attractors.

In the upper-well, Fig. 4, the orbits undergo significant qualitative changes. Sweeping the frequency down from $\Omega=10$, a branch of stable period-one orbits exists in the upper-well, this branch will be dubbed branch A. The stable period-one orbits lose stability via a supercritical period-doubling bifurcation at $\Omega=9.245$. Beyond the period-doubling bifurcation point, a stable period-two orbit coexists with an unstable period-one orbit. As shown in Fig. 5, the periodtwo orbit deforms as the frequency decreases and intersects the zero-velocity line only twice per period. The reason for this anomaly is that the response of the system at $2 \Omega$ continues to grow as the frequency decreases until it dominates the response at $\Omega=9.07$. 
Fig. 5 The phase portrait (a) and the time-history of the beam tip velocity (b) for a period-two orbit at $V_{\mathrm{AC}}=2 \mathrm{~V}$ and $\Omega=9$

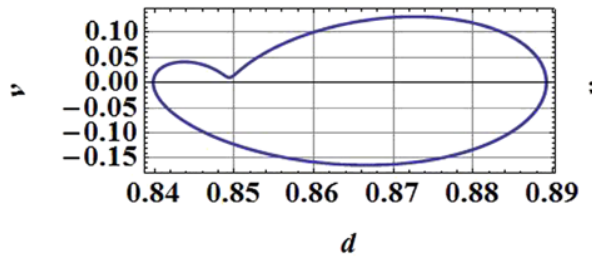

(a)

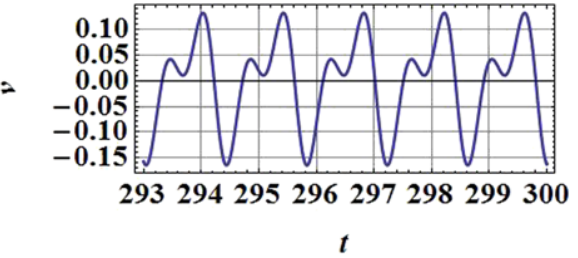

(b)

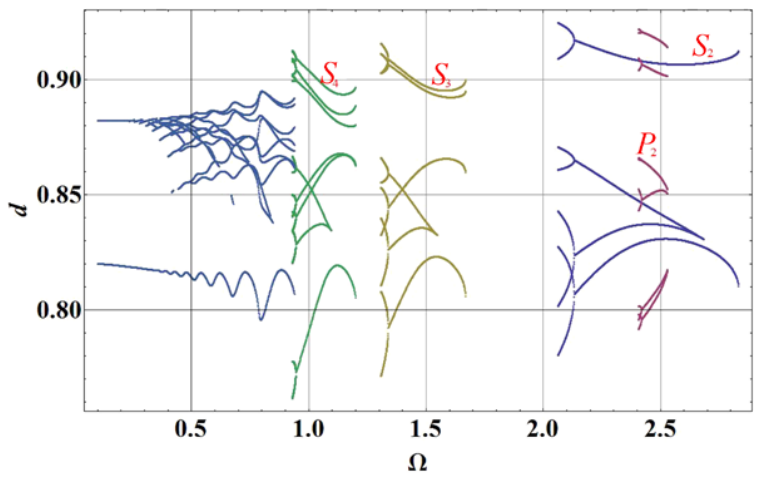

Fig. 6 (Color online) Bifurcation diagram of the super-harmonic orbits in the upper-well at $V_{\mathrm{AC}}=2 \mathrm{~V}$

As a result, each cycle sees only two velocity reversals and appears in the bifurcation diagram as two points instead of four. The stable period-two orbits undergo a cascade of period-doubling bifurcations culminating in chaos. Period four orbits appear at $\Omega=7.231$ and the chaotic attractors start at $\Omega=7.065$. This attractor, however, occupies a very small range in the frequency spectrum $\Omega=[7.055,7.076]$.

The branch of period-one unstable orbits originating from branch A regains stability through a subcritical period-doubling bifurcation at $\Omega=8.165$ where a new branch of stable orbits, dubbed branch $\mathrm{B}$, starts. This stable period-one orbit co-exists with an unstable period-two orbit born at the bifurcation point as well as the stable orbits of branch A described above. Sweeping the frequency down, branch B experiences a cascade of supercritical period-doubling bifurcations leading to chaos. Period-two orbits appear on the branch at $\Omega=4.589$, period-four orbits appear at $\Omega=4.374$, and chaos appears at $\Omega=4.344$.

At low excitation frequencies another set of branches exist in the upper-well, Fig. 3. The first branch of solutions, dubbed $S_{2}$ and shown in blue solid lines in Fig. 6, starts from a cyclic-fold bifurcation at $\Omega=2.833$ due to the super-harmonic resonance of order two. Figure 7 shows the phase portrait and the
FFT of an orbit on this branch at $\Omega=2.2$. The dominant peak in the FFT is present at twice the excitation frequency indicating a super-harmonic resonance of culminating in with chaos. Period-two orbits start at $\Omega=2.132$, period-four orbits start at $\Omega=2.061$, and chaos starts at $\Omega=2.05$.

A branch of period-two periodic orbits dubbed $P_{2}$, shown in red solid lines in Fig. 6, coexists with branch $S_{2}$. This branch appears out of a cyclic-fold bifurcation at $\Omega=2.53$. Figure 8 shows the phase portraits of the coexisting orbits on branches $S_{2}$ (solid blue line) and $P_{2}$ (dashed magenta line) at $\Omega=$ 2.5. While the orbits appear to intersect in the twodimensional projection of phase-space $(d, v)$, shown in Fig. 8, they do not intersect in the three-dimensional phase-space $\left(d, v, V_{s}\right)$. The orbits on this branch experience a cascade of period-doubling bifurcation; period-four orbits appear at $\Omega=2.419$ and chaos appears at $\Omega=2.045$. All of the chaotic attractors observed here were restricted to the upper-well.

Similarly, branches of super-harmonic orbits of order three $S_{3}$, shown in solid golden lines, and four $S_{4}$, shown in solid green lines, are observed in the neighborhoods of $\Omega \approx \frac{1}{3} \omega_{3}$ and $\Omega \approx \frac{1}{4} \omega_{3}$, Fig. 6 . These orbits appear through cyclic-fold bifurcations and disappear through a cascade of period-doubling bifurcations ending in chaos. The last branch of orbits, shown in Fig. 6 in solid blue lines, is born through a cyclic-fold bifurcation at $\Omega=0.94$. The orbits on this branch correspond to super-harmonic resonances of higher-order. Due to the quick succession of these super-harmonic resonances, the distinct branches of solutions merge in one branch with each super-harmonic resonance taking over from the next lowest as the frequency is decreased. No period-doubling bifurcations appear along this branch of solutions due to the weakness of these higher-order super-harmonic resonances.

In the lower-well, one branch of orbits, dubbed branch $\mathrm{C}$, exists throughout the frequency spectrum, Fig. 3, which undergoes dynamic amplification in the 
Fig. 7 The phase portrait (a) and the FFT (b) of an orbit at $V_{\mathrm{AC}}=2 \mathrm{~V}$ and $\Omega=2.2$

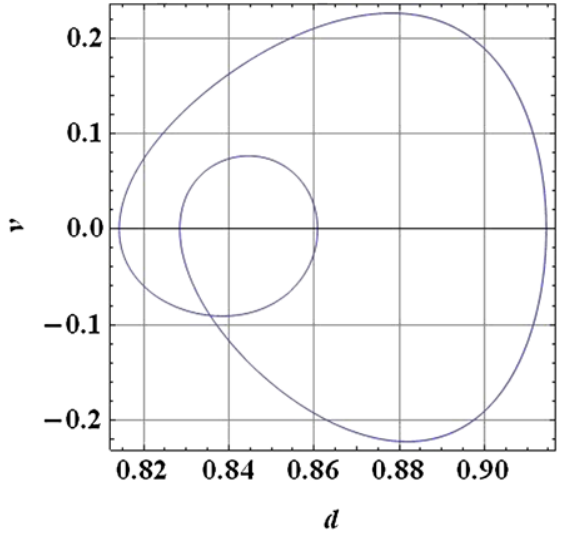

(a)

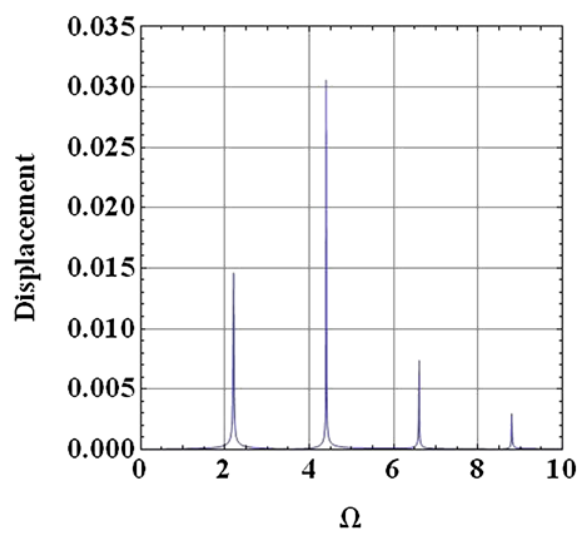

(b)

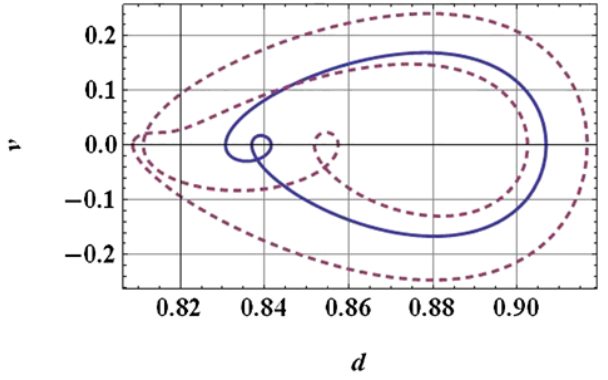

Fig. 8 (Color online) The phase portraits of the coexisting orbits at $V_{\mathrm{AC}}=2 \mathrm{~V}$ and $\Omega=2.5$

neighborhood of the natural frequency of the lower equilibrium position $\omega_{1}$.

\subsection{System response at $V_{\mathrm{AC}}=2.5 \mathrm{~V}$}

Figure 9 shows the bifurcation diagram for an $\mathrm{AC}$ amplitude $V_{\mathrm{AC}}=2.5 \mathrm{~V}$. In the upper-well, branches $\mathrm{A}$ and $\mathrm{B}$ are qualitatively similar to those appearing at $V_{\mathrm{AC}}=2 \mathrm{~V}$. Stable period-one orbits on branch A looses stability through a supercritical perioddoubling bifurcation at $\Omega=9.37$ and a cascade of period-doubling bifurcations takes place culminating with chaos at $\Omega=7.34$. Branch B starts at $\Omega=7.79$ with a subcritical period-doubling bifurcation giving birth to stable period-one orbits that loose stability through a supercritical period-doubling bifurcation at $\Omega=5.09$ followed by a cascade of period-doubling bifurcations ending with chaos at $\Omega=4.76$.

The chaotic region on branch B is larger than any of those realized in the upper-well for an AC amplitude of $V_{\mathrm{AC}}=2 \mathrm{~V}$. Figure 10 shows the bifurcation diagram of that chaotic region. The chaotic attractor experiences

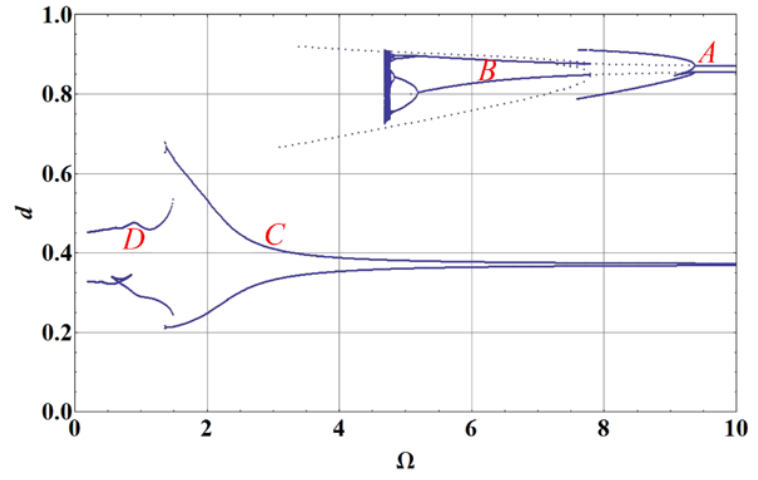

Fig. 9 The bifurcation diagram for $V_{\mathrm{AC}}=2.5 \mathrm{~V}$

an interior crisis at $\Omega=4.76$, after which it expands in size from a banded-attractor to a fully developed attractor filling most of the upper-well. The chaotic region experiences an exterior crisis at $\Omega=4.681$. Beyond the chaotic attractor, the stable manifold of the lower saddle re-injects the system response into the lower-well where it lands on the period-one orbits of branch C. It should be noted that this chaotic region appear in the vicinity of the natural frequency of the upper-well equilibrium position $\Omega \approx \omega_{3}$. None of the super-harmonic resonances of the upper-well observed at an $\mathrm{AC}$ amplitude of $V_{\mathrm{AC}}=2 \mathrm{~V}$ is present here.

The response in the lower-well, Fig. 9, is qualitatively similar to that for an excitation amplitude of $V_{\mathrm{AC}}=2 \mathrm{~V}$ except in the vicinity of the natural frequency of the lower equilibrium position $\Omega \approx \omega_{1}$. Branch $\mathrm{C}$ is split into two branches, Fig. 11, C at higher forcing frequencies and $\mathrm{D}$ at lower frequencies. As the frequency is swept down, the stable period-one orbits available on branch $\mathrm{C}$ experience a cascade of 


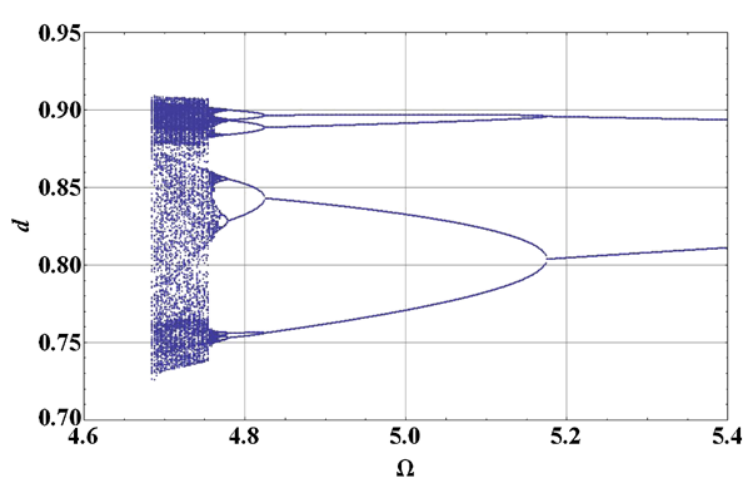

Fig. 10 The bifurcation diagram of the chaotic region on branch B at $V_{\mathrm{AC}}=2.5 \mathrm{~V}$

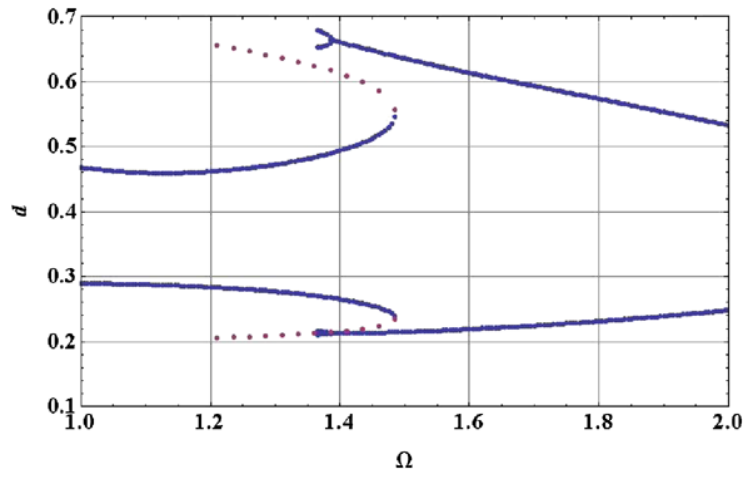

Fig. 11 Inset of Fig. 9

period-doubling bifurcations ending with chaos. The first period-doubling occurs at $\Omega=1.385$, the second at $\Omega=1.363$, the third at $\Omega=1.359$, and chaos appears at $\Omega=1.352$. Only the branch of period-two orbits, subsequent to the first period-doubling bifurcation, is shown due to the small span of the higherorder orbits. A homoclinic bifurcation was reported by Najar et al. [17] to interrupt an incomplete cascade of period-doubling bifurcations and to result in dynamic pull-in of an open-loop electrostatic resonator. In our case, the presence of the close-loop controller allows the cascade of period-doubling bifurcation to continue culminating in chaos due to a homoclinic entanglement. Sweeping the frequency down over the boundary of the chaotic region, the controller also re-injects the system response to the lower-well after it leaves the chaotic attractor to land on the period-one orbits of branch D.

Branch D starts through a cyclic-fold bifurcation at $\Omega=1.48$ that results in coexisting stable and unstable period-one orbits. These orbits co-exist with branch $\mathrm{C}$

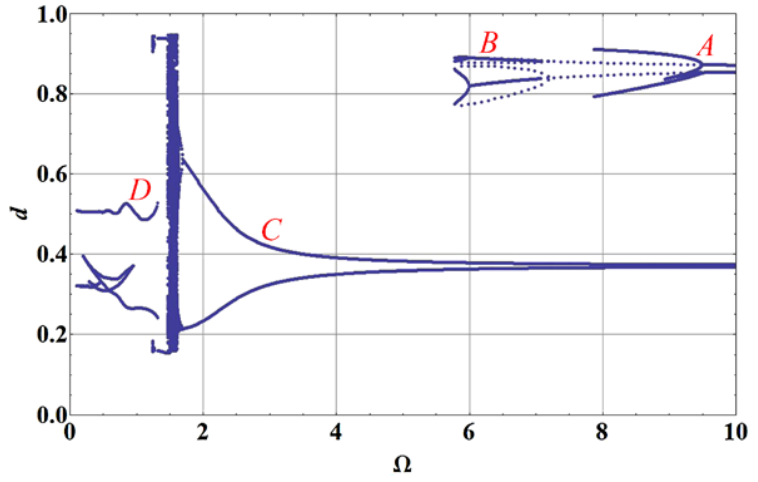

Fig. 12 The bifurcation diagram for $V_{\mathrm{AC}}=3 \mathrm{~V}$

in the interval $\Omega=[1.35,1.48]$ of the frequency spectrum. The orbits on branch D do not experience any qualitative change except for the appearance of superharmonic resonance of order two of the lower-well in the interval $\Omega=[0.55,0.86]$. The structure of the system response in the vicinity of the natural frequency of the lower-well $\Omega \approx \omega_{1}$, Fig. 11, indicates a softeningtype behavior.

\subsection{System response at $V_{\mathrm{AC}}=3 \mathrm{~V}$}

At this excitation amplitude, Fig. 12, minor changes occur in the upper-well branches while major changes occur in the lower-well branches. The only significant change in the upper-well is that the stable period-two orbits on branch B that originate from a supercritical period-doubling bifurcation at $\Omega=6$, meet a branch of unstable period-two orbits that originate from the subcritical period-doubling bifurcation at the right end of branch B at $\Omega=7.1$. Both of these orbits meet at $\Omega=5.78$ and vanish in a cyclic-fold bifurcation. The trend seen at $V_{\mathrm{AC}}=2.5 \mathrm{~V}$ continues at this forcing level with the span of branch B shrinking in the frequency spectrum. Branch A continues to demonstrate a cascade of period-doubling bifurcations ending in chaos was at $\Omega=7.7$.

Branch $\mathrm{C}$, in the lower-well, continues to experience a cascade of period-doubling bifurcations that ends in chaos. The bifurcations occur at higher forcing frequencies with period-two orbits appearing at $\Omega=1.69$, period-four orbits at $\Omega=1.643$, periodeight orbits at $\Omega=1.634$, and chaos at $\Omega=1.632$. The chaotic region, dubbed $C_{1}$, grows significantly, as shown in Fig. 13, to occupy a large frequency interval compared to the chaotic region at $V_{\mathrm{AC}}=2.5 \mathrm{~V}$. 


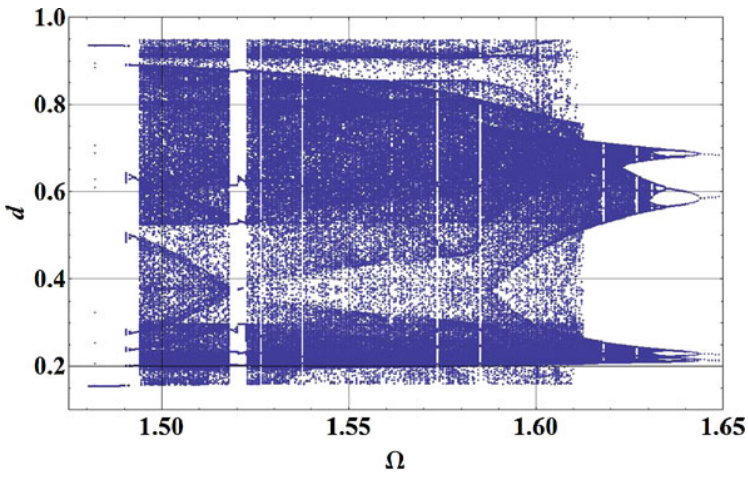

Fig. 13 The bifurcation diagram of the lower-well chaotic region at $V_{\mathrm{AC}}=3 \mathrm{~V}$

The chaotic region starts with a banded chaotic attractor that exists primarily in the lower-well with occasional excursions over the lower saddle. Figure 14 shows the phase portrait and the FFT of the chaotic attractor at $\Omega=1.615$. The broadband character of the FFT around the spike corresponding to the forcing frequency is a characteristic of chaotic motion [9]. At $\Omega=1.612$, the attractor experiences another interior crisis and expands abruptly to a fully-developed two-well chaotic attractor. Figure 15 shows the phase portrait and the FFT of the fully developed attractor at $\Omega=1.615$. The asymmetry and relative size of the potential wells can be seen in the phase portrait, Fig. 15a, as the chaotic attractor wanders to fill the upper- and lower-wells.

Figure 16 shows the maximum Lyapunov exponent of the attractors in the frequency interval $\Omega=$ $[1.45,1.65]$. The Lyapunov exponent corresponding to the phase angle $\lambda_{\theta}$ was found to be alway equal to zero. Excluding that exponent, the maximum Lyapunov exponent was found to take negative values for the periodic orbits preceding the chaotic region, to be zero at bifurcation points, and to be positive for
Fig. 14 The phase portrait (a) and the FFT (b) of a banded chaotic attractor at $V_{\mathrm{AC}}=3 \mathrm{~V}$ and $\Omega=1.615$

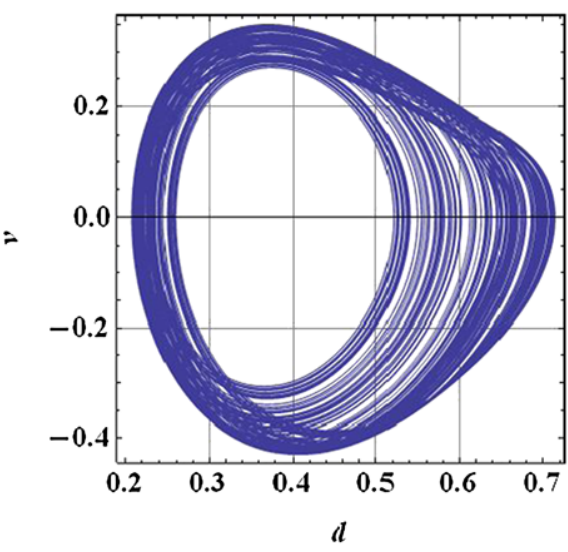

(a)

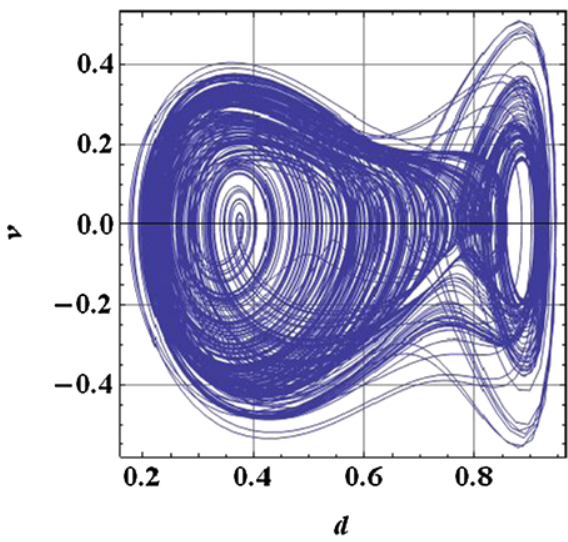

(a)

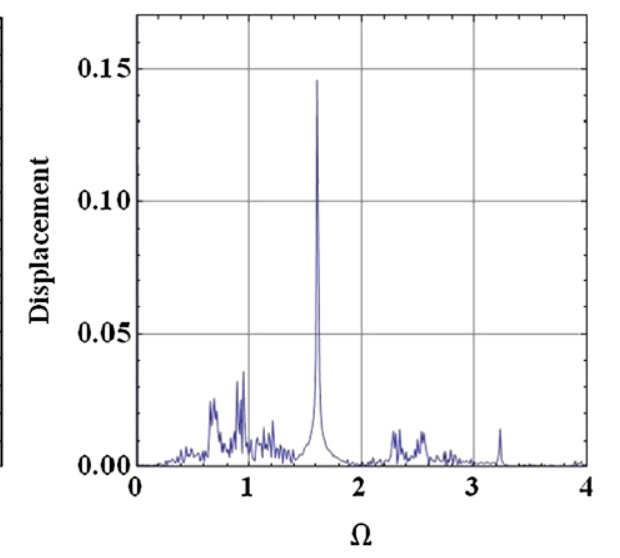

(b)

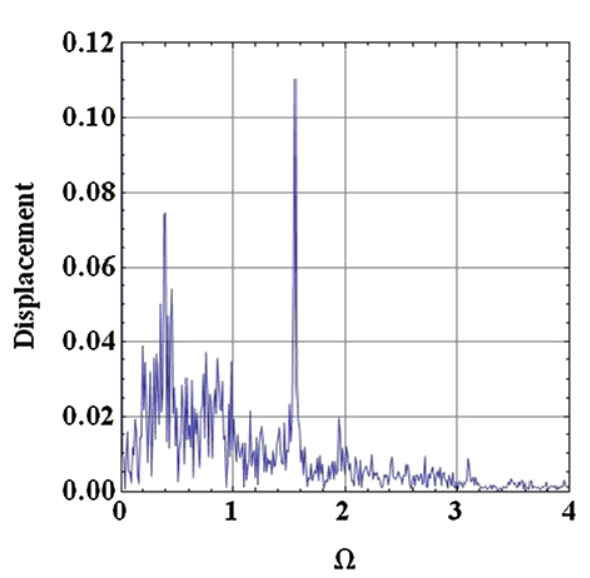

(b)
Fig. 15 The phase portrait (a) and the FFT (b) of a fully developed chaotic attractor at $\Omega=1.55$ and $V_{\mathrm{AC}}=3 \mathrm{~V}$ 


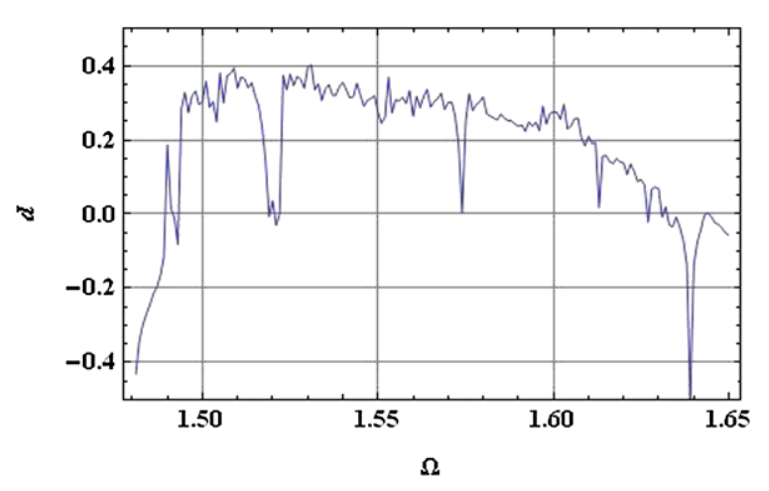

Fig. 16 The maximum Lyapunov exponent of the chaotic attractors at $V_{\mathrm{AC}}=3 \mathrm{~V}$

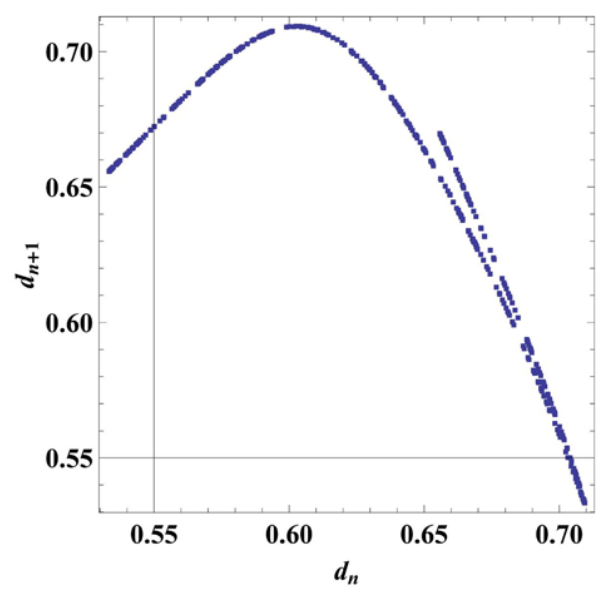

Fig. 17 The uni-modal return map of the chaotic attractor at $\Omega=1.615$

Fig. 18 Transient chaos (a) and the stable two-well orbit (b) at $V_{\mathrm{AC}}=3 \mathrm{~V}$ and $\Omega=1.442$ the chaotic attractors. The other two Lyapunov exponents were always negative. The figure shows that chaos starts at $\Omega=1.632$ and ends at $\Omega=1.494$. The magnitude of the maximum Lyapunov exponent grows with the increase in the size of the banded-chaotic attractor and settles in the neighborhood of $\lambda_{1} \approx 0.3$ as the fully-developed attractor sits in.

Periodic windows appear throughout the chaotic interval of the frequency spectrum indicated by downward spikes in the maximum Lyapunov exponent plot Fig. 16. A period-six window appears at $\Omega=1.627$, a period-five window appears at $\Omega=1.618$, periodfour windows appear at $\Omega=(1.585,1.574,1.522)$, and a period-three window appears at $\Omega=1.493$. This sequence of periodic windows was experimentally observed by Simoyi et al. [24] in the BelousovZhabotinskii chemical reaction. Figure 17 shows the return map constructed from the phase portrait of the chaotic attractor at $\Omega=1.615$ using a one-sided Poincaré section at $v=0$. The map is uni-modal in accordance with the theoretical finding of Metropolis et al. [25] that a system that going through a perioddoubling route to chaos will encounter dynamics similar to a uni-modal map which, in turn, demonstrates the aforementioned sequence of periodic windows.

The chaotic region ends with a boundary crisis at $\Omega=1.494$. Transient chaos appears beyond the crisis and the response settles down, over long-time, onto a two-well periodic orbit, Fig. 13. In this region, chaotic behavior is observed for more than 200 excitation periods before it settles onto the two-well periodic orbit. This orbit appears through a cyclic-fold bifurcation at $\Omega=1.493$. The phase portraits of the transient chaos and the two-well orbit at $\Omega=1.442$ are shown

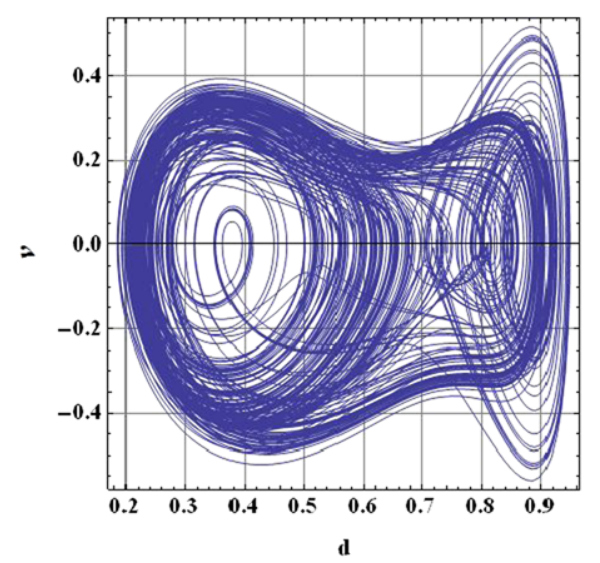

(a)

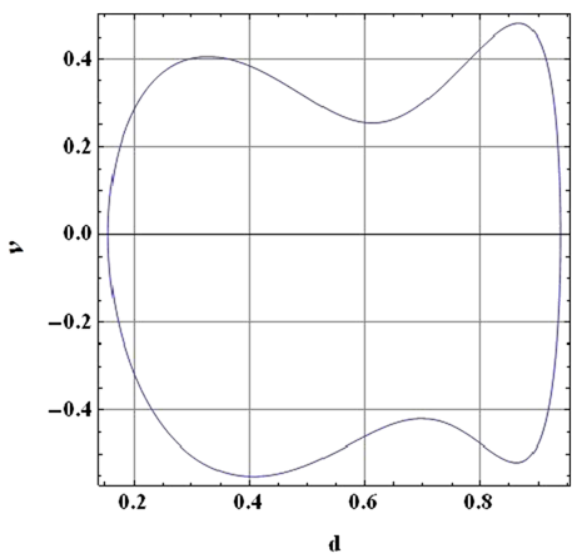

(b) 


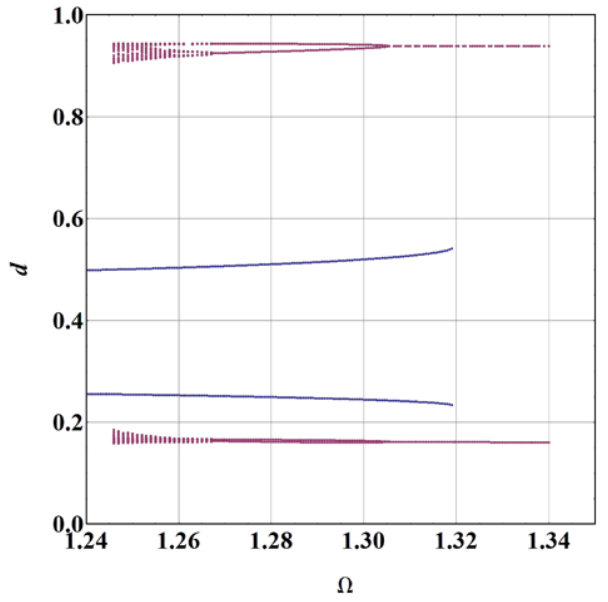

Fig. 19 (Color online) The coexistence of two-well branch of orbits and chaos with a stable one-well periodic orbit

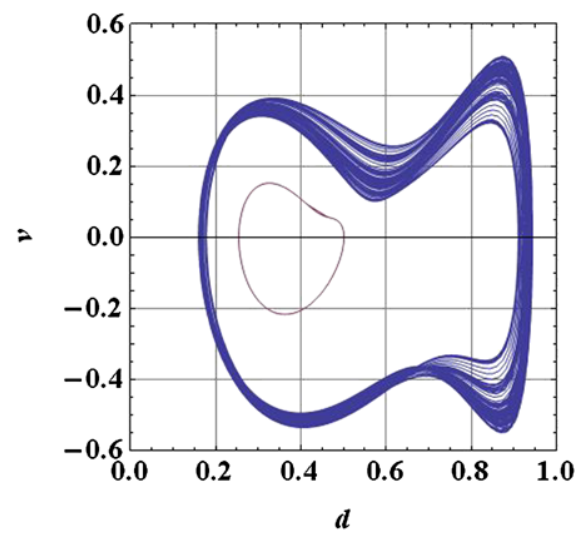

Fig. 20 Two-well banded chaos co-existing with a one-well stable orbit at $\Omega=1.25$ and $V_{\mathrm{AC}}=3 \mathrm{~V}$

in Fig. 18. The figure indicates that the two-well orbit resides completely outside the lower- and upperwells. Sweeping the frequency down, the two-well orbit experiences a cascade of period-doubling bifurcations and ends at $\Omega=1.26$ with a region of banded chaos dubbed $C_{2}$. The chaotic region ends in a boundary crisis and transient chaos appears in its place.

Branch D appears at $\Omega=1.319$ through a cyclicfold bifurcation and evolves over the frequency domain in a similar manner to that seen at lower excitation amplitudes. In the frequency interval [1.242, 1.319], branch D, shown in blue lines in Fig. 19, coexists with the banded two-well chaotic region, shown in red lines in the same figure. In Fig. 20, we show phase portraits of the coexisting stable one-well orbit

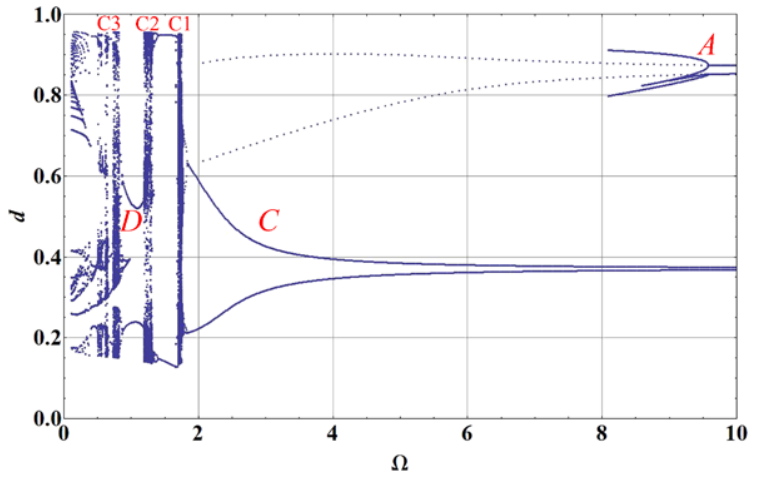

Fig. 21 The bifurcation diagram for $V_{\mathrm{AC}}=3.5 \mathrm{~V}$

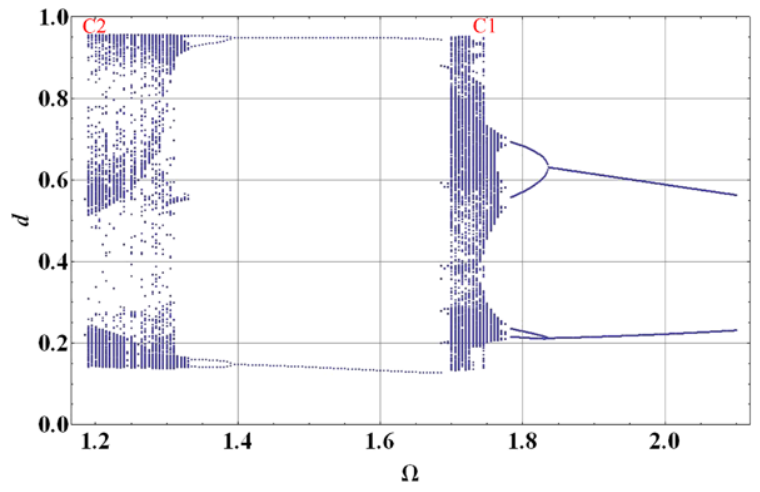

Fig. 22 Bifurcation diagram for the chaotic regions at $V_{\mathrm{AC}}=3.5 \mathrm{~V}$

on branch D and two-well banded chaotic attractor at $\Omega=1.25$.

\subsection{System response at $V_{\mathrm{AC}}=3.5 \mathrm{~V}$}

Figure 21 shows the bifurcation diagram at an excitation amplitude of $V_{\mathrm{AC}}=3.5 \mathrm{~V}$. The frequency interval of branch A continues to shrink, while the evolution of the attractors on the branch over the frequency spectrum continue in a similar manner to that seen at lower excitation levels. The branch of unstable period-one orbits emerging from branch A does not regain stability, and as a result, branch B disappears from the system response.

The cascade of period-doubling bifurcations and the chaotic attractors on branch $\mathrm{C}$ appear at higher excitation frequencies. The first period-doubling bifurcation takes place at $\Omega=1.835$ and chaos starts at $\Omega=1.775$. The frequency interval occupied by chaotic region $C_{1}$ shrinks, while those occupied by the 
Fig. 23 The phase portrait (a) and the FFT (b) of a two-well chaotic attractor at $V_{\mathrm{AC}}=3.5 \mathrm{~V}$ and $\Omega=1.2$

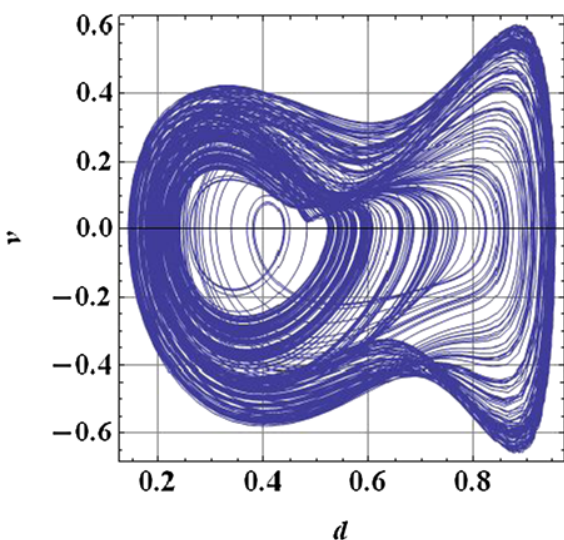

(a)

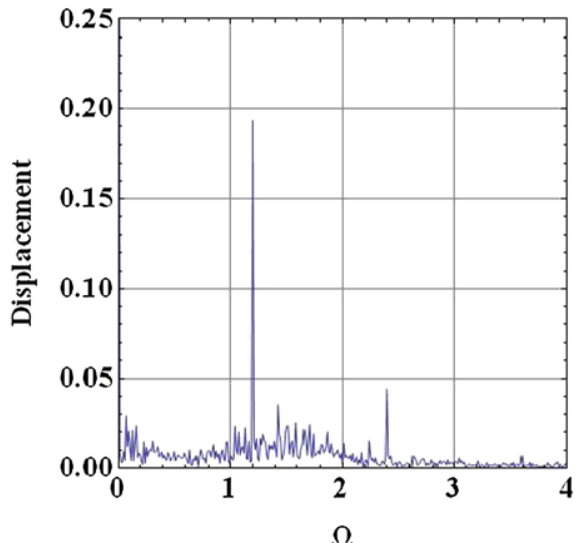

(b)

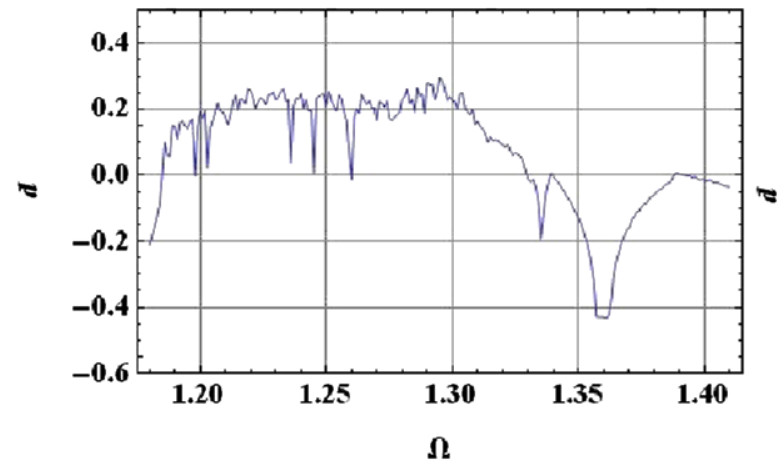

(a)

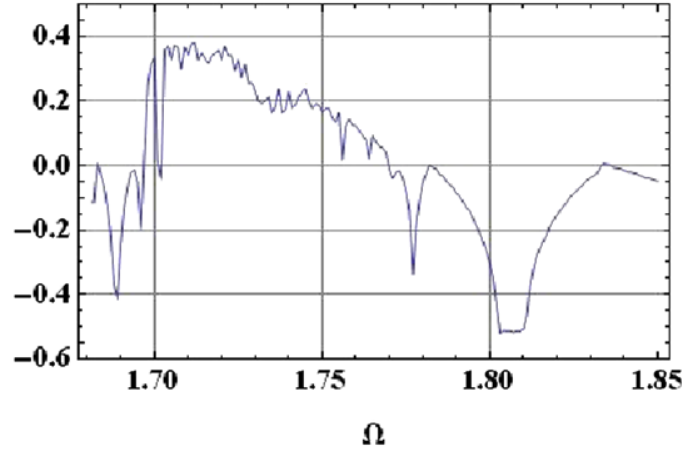

(b)

Fig. 24 The maximum Lyapunov exponent of the chaotic attractors at $V_{\mathrm{AC}}=3.5 \mathrm{~V}$

two-well orbit and the two-well chaotic region $C_{2}$ expand. The bifurcation diagram of chaotic regions $C_{1}$ and $C_{2}$ is shown in Fig. 22. As the frequency interval of chaotic region $C_{2}$ increases, the banded chaotic attractor evolving over the right-side of the region undergoes an internal crisis and changes abruptly to a fully developed chaotic attractor filling the region in phase-space where the two-well orbit existed. Figure 23 shows the phase portrait and the FFT of a fully developed two-well chaotic attractor in region $C_{2}$ at $\Omega=1.2$. We note that these attractors unlike those of region $C_{1}$ do not exhibit any oscillation in the upperwell alone.

The maximum Lyapunov exponent, excluding $\lambda_{\theta}$, for chaotic regions $C_{1}$ and $C_{2}$ is shown in Fig. 24a and Fig. 24b, respectively. From the FFT and the Lyapunov exponent plots, it can be seen that the fully developed two-well chaotic attractors of regions $C_{1}$ are more active than those of region $C_{2}$. The maximum Lyapunov exponent for region $C_{1}$ is $\lambda_{1} \approx 0.4$ and for region $C_{2}$ is $\lambda_{1} \approx 0.3$.

Branch D appears at $\Omega=1.185$ out of a cyclicfold bifurcation, Fig. 21. It starts with stable lowerwell period-one orbits. Unlike the case at lower excitation amplitudes, branches $\mathrm{C}$ and $\mathrm{D}$ do not co-exist. As the frequency is increased past the cyclic fold bifurcation, intermittency occurs with the resonator visiting the ghost of the period-one orbit frequently and bursting out into the upper-well occasionally. Figure 25 shows the phase portrait and time-history of the intermittent system response at $\Omega=1.186$. The ghost of the period-one orbit can be seen in the lowerwell while the bursts span the two wells. Therefore, chaotic region $C_{2}$ at this excitation amplitude changes structure to appear through a period-doubling route to chaos in a frequency down-sweep and an intermittency of type-I route to chaos in a frequency upsweep. 
Fig. 25 Phase portrait (a) and time-history of $d$ (b) of the resonator response during intermittent behavior at $V_{\mathrm{AC}}=3.5 \mathrm{~V}$ and $\Omega=1.186$

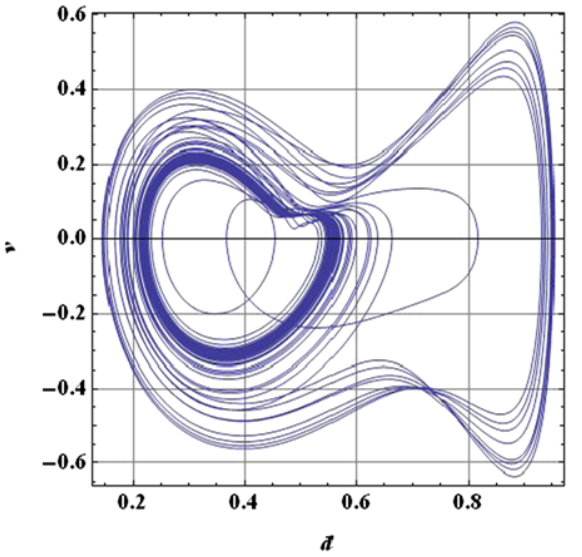

(a)

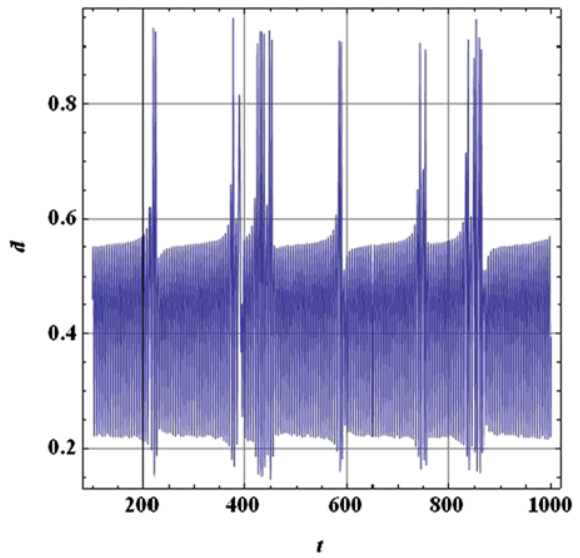

(b)
As the excitation frequency decreases, the stable period-one orbits on branch $\mathrm{D}$ experience a cascade of period-doubling bifurcations ending in chaos at $\Omega=0.825$. Chaos persists over the frequency range $\Omega=[0.729,0.825]$, Fig. 26, this chaotic region will be dubbed $C_{3}$. The increase in the response amplitude and the concomitant period-doubling cascade and chaos occur as the frequency down-sweep approaches the super-harmonic resonance of order two of the lower-well $\Omega \approx \frac{1}{2} \omega_{1}$. Similar to chaotic region $C_{2}$, region $C_{3}$ appears through an intermittency of type-I in a frequency up-sweep. Beyond the cyclic-fold bifurcation at the left boundary of chaotic region $C_{3}$, appears a branch of two-well super-harmonic orbits of order three. Higher-order super-harmonic resonances of the lower-well $\Omega \approx \frac{1}{n} \omega_{1}$ continue to appear as the frequency down-sweep proceeds. They have similar structure to that of the super-harmonic resonance of order two and their orbits are alternately lower-well for odd-numbered super-harmonic resonances and twowell for even-numbered super-harmonic resonances. Typical orbits of the supper harmonic resonances of orders two to five are shown in Fig. 27. The orbits of the super-harmonic resonances of order two and four are seen to exist completely in the lower-well, while those of order three and five span the two wells. Distinct chaotic regions can be seen within the superharmonic resonances of order three and four in Fig. 26.

\subsection{System response at $V_{\mathrm{AC}}=4 \mathrm{~V}$}

The evolution of the system response at this excitation amplitude is qualitatively similar to that at $V_{\mathrm{AC}}=$

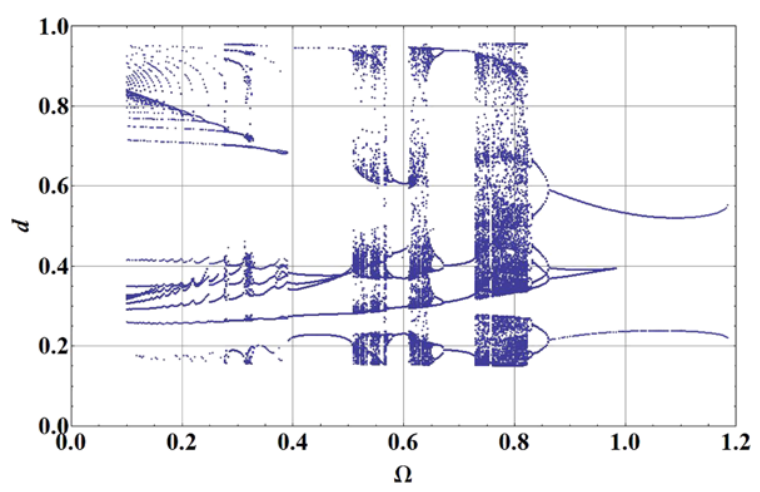

Fig. 26 The bifurcation diagram of the superharmonic resonances at $V_{\mathrm{AC}}=3.5 \mathrm{~V}$

3.5 $\mathrm{V}$ except for the frequency interval below the natural frequency of the lower equilibrium position $\omega_{1}=1.98$. Figure 28 shows the bifurcation diagram of the system response in that interval. Similar to the case at $V_{\mathrm{AC}}=3.5 \mathrm{~V}$, the cascade of period-doubling bifurcation and chaotic attractors on branch $\mathrm{C}$ appear at higher forcing frequencies, the frequency interval of chaotic region $C_{1}$ shrinks to $\Omega=[1.883,1.837]$, and the frequency interval of chaotic region $C_{2}$ expands to $\Omega=[1.372,1.051]$. However, the fully developed chaotic attractors in region $C_{1}$ become primarily onewell attractors. Further, the two-well period-one orbit starts at $\Omega=1.85$, thereby co-existing with a part of chaotic region $C_{1}$.

Similar to before, branch D starts with a cyclicfold bifurcation just after the chaotic region $C_{2}$. The frequency range over which the stable period-one orbits on branch $\mathrm{D}$ exists shrank and the chaotic regions $C_{2}$ and $C_{3}$ became very close and can be re- 
A. Seleim et al.

Fig. 27 The phase portraits of the super-harmonic orbits of order (a) two $\Omega=0.87$, (b) three $\Omega=0.68$, (c) four $\Omega=0.58$, and (d) five $\Omega=0.44$ at $V_{\mathrm{AC}}=3.5 \mathrm{~V}$

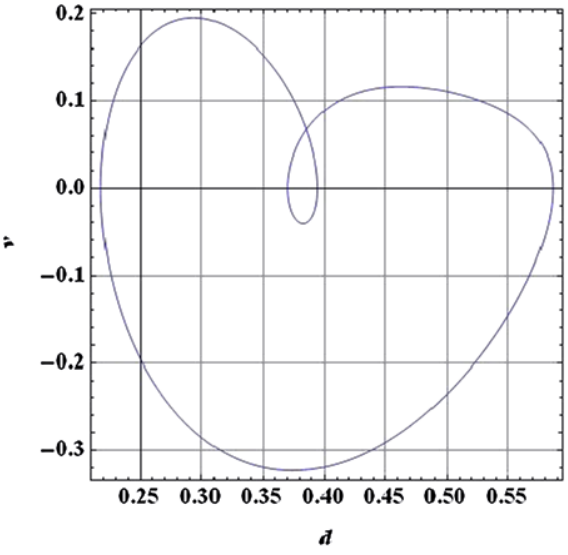

(a)

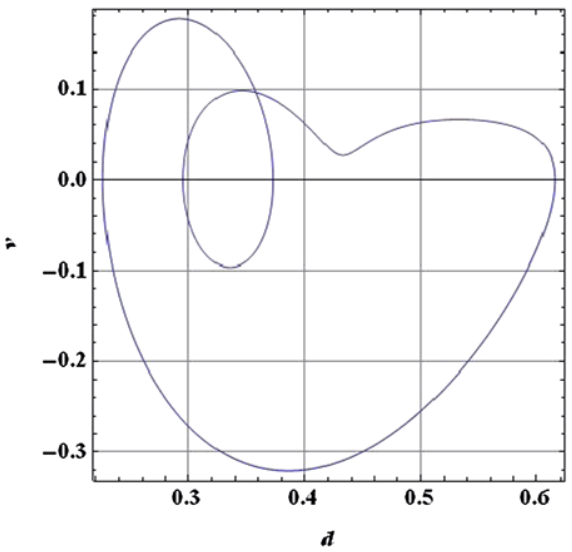

(c)

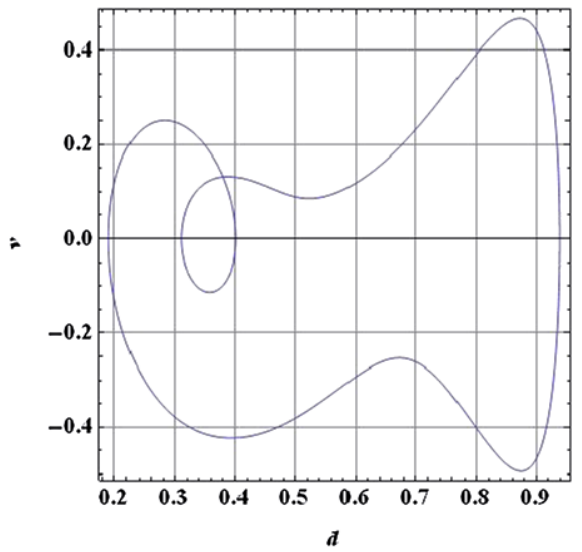

(b)

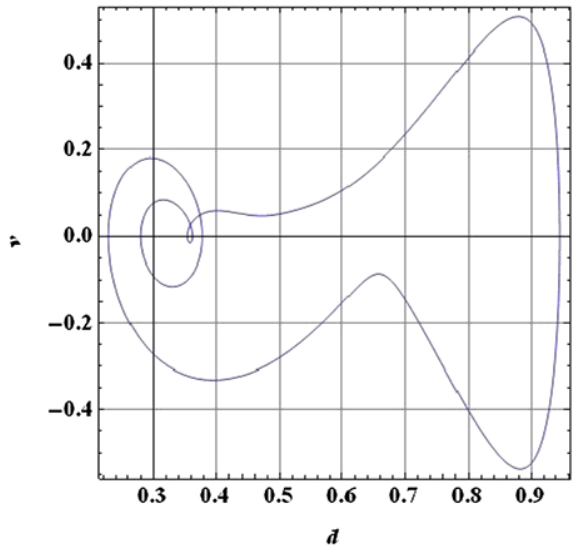

(d)
Fig. 28 The bifurcation diagram for the chaotic regions at $V_{\mathrm{AC}}=4 \mathrm{~V}$

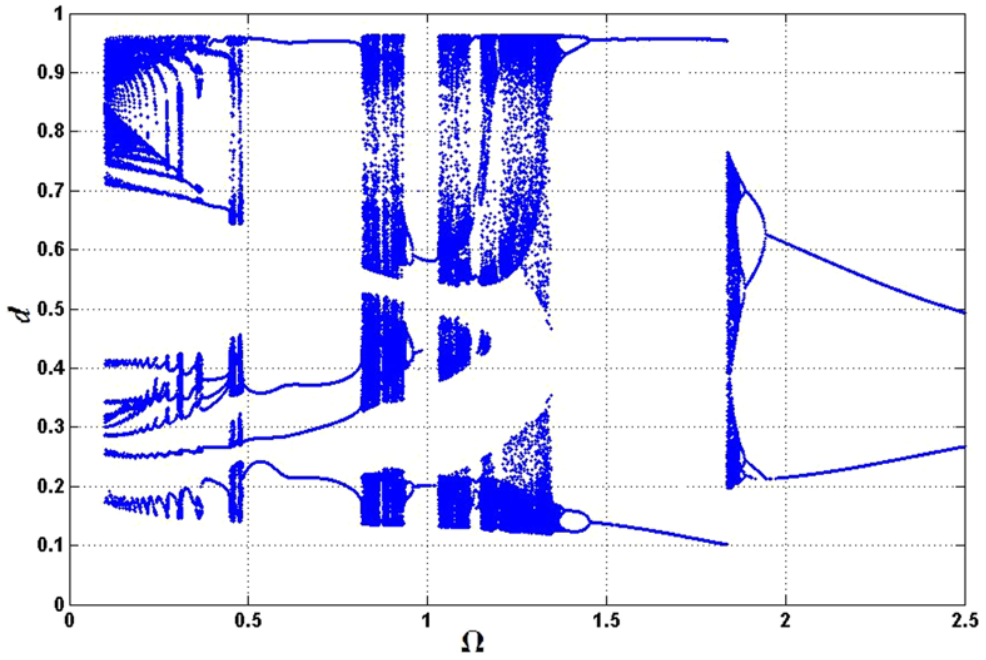


garded as one chaotic region. Super-harmonic resonances of the lower equilibrium natural frequency $\omega_{1}$ continue to appear at forcing frequencies below $\Omega=0.5$. This bifurcation diagram is appears as Towfighian et al. [20] using a stroboscopic Poincaré section.

\subsection{Stability of the chaotic attractors}

As mentioned in the Introduction, this chaotic oscillator is designed as a platform for a mass sensor. The basins of attraction of the chaotic attractors are studied to evaluate how realistic are the chances of landing on the attractor in practical applications. The basins of attraction of selected chaotic attractors in regions $C_{1}$ and $C_{2}$ were generated to investigate the longterm behavior of those attractors. The figures, representing the basin of attraction, were constructed by holding $V_{s}[0]=0$ and dividing $(d, v)$ phase-space into a $(300 \times 300)$ grid of initial conditions. Long time integration was carried out from each grid point for one hundred excitation periods to assure that transients have decayed and the system has settled on an orbit. The maximum position in the last period was recorded. The basin of attraction was then plotted as a colored grid where the color intensity decreases with the magnitude of the recorded position $d$. The color changes from dark blue for $d=0$ to light hue for $d=1$. In cases of pull-in, the value of $d$ is assigned equal to 1 .

First, we test our basin construction technique using the three stable co-existing orbits at $V_{\mathrm{AC}}=2 \mathrm{~V}$ and $\Omega=7.5$, Fig. 3. The basin of attraction for this forcing level is shown in Fig. 29. The three stable orbits available are a period-two orbit on branch A with a maximum position of $d=0.910$, a periodone orbit on branch $\mathrm{B}$ with a maximum position of $d=0.875$, and a period-one orbit on branch $\mathrm{C}$ with a maximum position of $d=0.374$. The maximum of the last two excitation periods was recorded to accommodate the period-two orbit. Initial conditions terminating in the upper-well orbit on branch A $(d=0.910)$ and pull-in $(d=1)$ appear in a light hue. The upperwell orbit on branch B $(d=0.875)$ appears in a darker hue. The lower-well orbit on branch $\mathrm{C}(d=0.374)$ appears in blue. The basin of attraction shows the separation of initial conditions into distinct strips as the stable and unstable manifolds of the saddles deform.

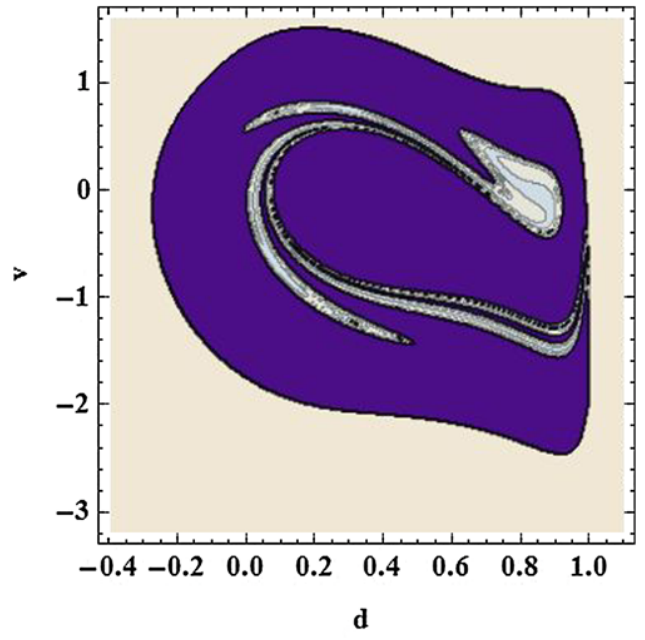

Fig. 29 (Color online) The basin of attraction of the orbits at $V_{\mathrm{AC}}=2 \mathrm{~V}$ and $\Omega=7.5$

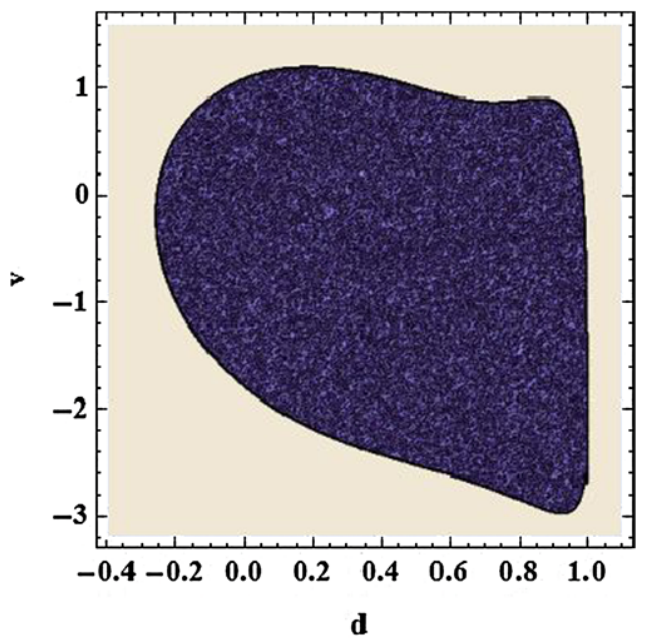

Fig. 30 (Color online) The basin of attraction of a chaotic attractor at $V_{\mathrm{AC}}=3 \mathrm{~V}$ and $\Omega=1.62$

Figure 30 shows the basin of attraction for a banded primarily one-well chaotic attractor in region $C_{1}$ at $V_{\mathrm{AC}}=3 \mathrm{~V}$ and $\Omega=1.62$ (similar to that shown in Fig. 14a). Figure 31 shows the basin of attraction for a fully developed two-well chaotic attractor in regions $C_{2}$ at $V_{\mathrm{AC}}=3.5 \mathrm{~V}$ and $\Omega=1.2$ (shown in Fig. 23a). In both cases, the initial conditions inside the potential wells and in their immediate vicinity land on the chaotic attractors as indicated by the well mixed character of the color map. This is a characteristic $\mathrm{n}$ of the chaotic attractor mixing actio. Initial conditions fur- 


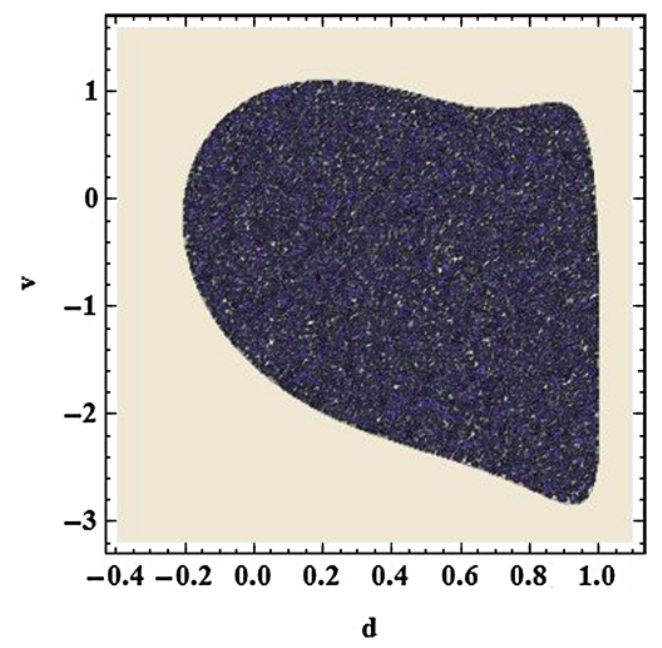

Fig. 31 (Color online) The basin of attraction of a chaotic attractor at $V_{\mathrm{AC}}=3.5 \mathrm{~V}$ and $\Omega=1.2$

ther away from the wells go to pull-in. Therefore, the basins of attraction of both chaotic attractors encompass a wider region in phase-space than the attractors themselves, Figs. 14 and 23. The presence of a buffer zone where initial conditions in the vicinity of the attractor land on them ensures their stability under external disturbances.

\section{Conclusion}

At low excitation levels, the response of the electrostatic actuator shows one branch of solutions in the lower potential well and two branches of solutions in the upper potential well. As the excitation level increases, lower frequency branch disappears from the upper well, while the higher frequency branch shrinks. Meanwhile, two branches of solutions appear and persist in the lower well. Superharmonic resonances were observed in both wells. In the upper-well, super-harmonic resonances were observed at low excitation levels, namely independent branches of upper-well super-harmonic resonances of order two, three, and four. All of these branches start from a cyclic-fold bifurcation and end in chaos through cascade of period-doubling bifurcations. Lower-well super-harmonic resonances were observed at higher excitation levels. These resonances lead to the appearance of distinct low frequency chaotic regions.
In agreement with previous experimental and analytical reports of chaos in electrostatic MEMS [14, 15], all the chaotic regions we found appeared through period-doubling routes to chaos. We found that chaotic region $C_{1}$ disappears through a boundary crisis to be replaced with transient chaos at lower excitation frequencies. Further, for higher excitation levels $V_{\mathrm{AC}} \geq 3.5 \mathrm{~V}$, we found that chaotic region $C_{2}$ and the chaotic regions within super-harmonic resonances appear through an intermittency of type-I route to chaos in frequency up-sweep.

This study provides qualitative insight into the usability of the actuation parameter space for the various uses of electrostatic micro-beam actuators. Orbits in the upper-well, along branches $\mathrm{A}$ and $\mathrm{B}$, are small in size. They encounter zones in the frequency domain where multi-valuedness occur and two orbits co-exist. Further, the orbits come very close to pull-in where the electrostatic force grows very fast, thereby undermining the orbit stability to external disturbances. Therefore, we conclude that it is not suitable to operate periodic resonators along these branches.

The periodic orbits appearing along branch $\mathrm{C}$ provide the largest possible dynamic amplification in the parameter space. Therefore, it is desirable to operate periodic resonators along this branch provided that the amplitude of $\mathrm{AC}$ excitation is maintained below the threshold for the appearance of chaos, $V_{\mathrm{AC}}=$ $2.5 \mathrm{~V}$ in this case. The regions in the parameter space where super-harmonic resonances appear provide a good opportunity to realize large period periodic oscillators. The lower-well super-harmonic resonances provide better dynamic amplification, and therefore less demands for voltage, than the upper-well superharmonic resonances. Again, the amplitude of excitation has to be limited to a value below the threshold where chaos appears with super-harmonic resonances; $V_{\mathrm{AC}}=3 \mathrm{~V}$ in this case.

The availability of steady-state chaos over a wide range in parameter space is a requirement for the use of chaotic resonators in sensing applications. Steadystate chaotic attractors were found at all the excitation levels considered. The chaotic regions in the upperwell were found to cover narrow bands in the frequency spectrum, which render them unsuitable for sensing purposes. On the other hand, chaotic regions $C_{1}, C_{2}$, and $C_{3}$ were found to cover wider bands in the frequency spectrum and are, thus, more suitable for sensing purposes. 
The bandwidth of chaotic region $C_{1}$ increased as the excitation amplitude is increased from $V_{\mathrm{AC}}=$ $2.5 \mathrm{~V}$ to $V_{\mathrm{AC}}=3 \mathrm{~V}$ then decreased as the excitation amplitude is continued to increase to $V_{\mathrm{AC}}=4 \mathrm{~V}$. The maximum bandwidth of this chaotic region exists in the neighborhood of $V_{\mathrm{AC}}=3 \mathrm{~V}$. Similarly, chaotic region $C_{3}$, appearing for the first time at $V_{\mathrm{AC}}=3.5 \mathrm{~V}$, saw its bandwidth decreasing at $V_{\mathrm{AC}}=3 \mathrm{~V}$, which indicates that its maximum bandwidth exists in the neighborhood of $V_{\mathrm{AC}}=3.5 \mathrm{~V}$. On the other hand, the bandwidth of chaotic region $C_{2}$ increased consistently from $V_{\mathrm{AC}}=3 \mathrm{~V}$ to $V_{\mathrm{AC}}=4 \mathrm{~V}$. The chaotic attractors of region $C_{1}$ have the advantages of spending more time away from the upper saddle, which lowers the possibility of encountering pull-in, and being more active than the chaotic attractors in regions $C_{2}$ and $C_{3}$. On the other hand, the maximum bandwidth of chaotic regions $C_{2}$ and $C_{3}$ is larger than that of region $C_{1}$. The primarily one-well part of chaotic region $C_{1}$ seems to be most suited for sensing purposes since that region had the highest rate of change of the maximum Lyapunov exponent.

Acknowledgements The authors would like to thank the Canadian Foundation for Innovation (CFI) and the Natural Sciences and Engineering Research Council of Canada (NSERC) for their support.

\section{Appendix A: Procedure for constructing bifurcation diagrams}

The following procedure was used to construct the bifurcation diagrams shown in this paper:

1. At an excitation frequency $\Omega$ away from the stable natural frequencies $\omega_{1}$ and $\omega_{3}$, long time integration was performed until the system settled on a stable period-one orbit. The numerical integration was carried out in Mathematica using the Modified Adams method [26].

2. The states at an arbitrary point on the orbit were recorded and used as an initial guess $\left(d_{o}, v_{o}, V_{s o}\right)$ for the shooting method. The shooting method turns the problem of finding the closed orbits to a boundary-value problem where the initial and final points on the orbit, after one period, are matched. It then uses an initial-value solver to integrate the system equations for a period and interprets the difference between the initial and final point as an error to be corrected iteratively.
3. The shooting method was implemented by integrating the system equations for a period $T=\frac{2 \pi}{\Omega}$. The difference between the initial guess and the terminal point $\left(d_{f}, v_{f}, V_{s f}\right)$ is then used to correct the initial guess until the magnitude of the Euclidean norm of the error vector is less than $10^{-3}$.

4. The monodromy matrix of the orbit was obtained from numerical integration of the system of equations, augmented with the system of linearized perturbations around the orbit, for a period starting from an initial condition on the converged orbit [9].

5. The Floquet multipliers (the Eigenvalues of the monodromy matrix) were calculated. Floquet theory [9] was then used to determine the stability of the orbits, based on the values of those multipliers, and to determine the type of the bifurcation.

6. The excitation frequency was incremented to a larger or smaller value depending on the frequency sweep direction and a point on the converged orbit at the previous frequency was used as the initial guess for the shooting method.

7. Steps 3-6 were repeated until the tracked orbit lost stability.

- Where stability was lost through a period-doubling bifurcation, steps 3 to 6 were repeated and the period of integration was doubled.

- Where stability was lost through a cyclic-fold bifurcation, steps 1 to 6 were repeated while searching for a new set of orbits.

8. Unstable orbits were tracked where relevant.

9. For every orbit, the intersections with the zerovelocity line $(v=0)$ were recorded. A period-one orbit is represented by two points, a period-two orbit is represented by 4 points, etc.

10. The bifurcation diagram was then constructed by stacking the Poincare sections as a function of the excitation frequency. Since the increment in frequency is small, these discrete points appear as continuous lines. For unstable orbits, one point every ten points was plotted in order to appear as dotted lines.

11. Because motion within a chaotic attractor is aperiodic, the shooting method fails there. Instead, long-time integration was used and the intersections of the flow with the zero-velocity line were recorded for one thousand periods of excitation. The intersections were recorded after at least one 
hundred periods of integration to allow for the transient response to decay.

\section{Appendix B: Algorithm for calculating Lyapunov exponents}

The Lyapunov exponents of the four-dimensional phase-space were calculated using the following procedure [9]:

1. The system equations (5)-(8) were augmented with the system of linearized perturbations around the orbit $[y(t)]$, and integrated for a period of time $T_{f}$. The initial conditions of the dynamic system were chosen arbitrarily while the initial conditions of the linearized perturbations $[y(0)]$ were set equal to the identity matrix.

2. The vectors $\mathbf{y}_{1}\left(T_{f}\right), \mathbf{y}_{2}\left(T_{f}\right), \mathbf{y}_{3}\left(T_{f}\right)$, and $\mathbf{y}_{4}\left(T_{f}\right)$ were obtained from the columns of $\left[y\left(T_{f}\right)\right]$.

3. A new set of initial perturbation vectors was obtained by ortho-normalization through the GrahamSchmidt procedure:

$$
\begin{aligned}
& \hat{\mathbf{y}}_{1}=\frac{\mathbf{y}_{1}\left(T_{f}\right)}{\left\|\mathbf{y}_{1}\left(T_{f}\right)\right\|} \\
& \hat{\mathbf{y}}_{2}=\frac{\mathbf{y}_{2}\left(T_{f}\right)-\left(\mathbf{y}_{2}\left(T_{f}\right) \cdot \hat{\mathbf{y}}_{1}\right) \hat{\mathbf{y}}_{1}}{\left\|\mathbf{y}_{2}\left(T_{f}\right)-\left(\mathbf{y}_{2}\left(T_{f}\right) \cdot \hat{\mathbf{y}}_{1}\right) \hat{\mathbf{y}}_{1}\right\|} \\
& \hat{\mathbf{y}}_{3}=\frac{\mathbf{y}_{3}\left(T_{f}\right)-\left(\mathbf{y}_{3}\left(T_{f}\right) \cdot \hat{\mathbf{y}}_{2}\right) \hat{y}_{2}-\left(\mathbf{y}_{3}\left(T_{f}\right) \cdot \hat{\mathbf{y}}_{1}\right) \hat{\mathbf{y}}_{1}}{\left\|\mathbf{y}_{3}\left(T_{f}\right)-\left(\mathbf{y}_{3}\left(T_{f}\right) \cdot \hat{\mathbf{y}}_{2}\right) \hat{\mathbf{y}}_{2}-\left(\mathbf{y}_{3}\left(T_{f}\right) \cdot \hat{\mathbf{y}}_{1}\right) \hat{\mathbf{y}}_{1}\right\|}
\end{aligned}
$$

$$
\hat{\mathbf{y}}_{4}=
$$

$\frac{\mathbf{y}_{4}\left(T_{f}\right)-\left(\mathbf{y}_{4}\left(T_{f}\right) \cdot \hat{\mathbf{y}}_{3}\right) \hat{\mathbf{y}}_{3}-\left(\mathbf{y}_{4}\left(T_{f}\right) \cdot \hat{\mathbf{y}}_{2}\right) \hat{\mathbf{y}}_{2}-\left(\mathbf{y}_{4}\left(T_{f}\right) \cdot \hat{\mathbf{y}}_{1}\right) \hat{\mathbf{y}}_{1}}{\left\|\mathbf{y}_{4}\left(T_{f}\right)-\left(\mathbf{y}_{4}\left(T_{f}\right) \cdot \hat{\mathbf{y}}_{3}\right) \hat{\mathbf{y}}_{3}-\left(\mathbf{y}_{4}\left(T_{f}\right) \cdot \hat{\mathbf{y}}_{2}\right) \hat{\mathbf{y}}_{2}-\left(\mathbf{y}_{4}\left(T_{f}\right) \cdot \hat{\mathbf{y}}_{1}\right) \hat{\mathbf{y}}_{1}\right\|}$

where $(a . b)$ denotes the dot product.

4. The Lyapunov exponents were calculated as

$$
\lambda_{i}=\frac{1}{r T_{f}} \sum_{k=1}^{r} \ln N_{i}^{k}
$$

where $N$ is the norm in the denominator of $\hat{\mathbf{y}}_{i}$, the subscript $i$ refers to the $i$ th vector, the superscript $k$ refers to the iteration number, and $r$ is the total number of iterations.

5. The augmented system was integrated again for a period $T_{f}$ where the state values and $\hat{\mathbf{y}}_{i}$ vectors at $t=T_{f}$ were used as initial conditions.
6. Steps 2-5 were repeated until the values of the Lyapunov exponents asymptotically converged.

\section{References}

1. Lang, H.P., Berger, R., Battiston, F., Ramseyer, J.P., Meyer, E., Andreoli, C., Brugger, J., Vettiger, P., Despont, M., Mezzacasa, T., Scandella, L., Güntherodt, H.-J., Gerber, Ch., Gimzewski, J.K.: A chemical sensor based on a micromechanical cantilever array for the identification of gases and vapors. Appl. Phys. A, Mater. Sci. Process. 66, 61-64 (1998)

2. Cherian, S., Gupta, R.K., Mullin, B.C., Thundat, T.: Detection of heavy metal ions using protein-functionalized microcantilever sensors. Biosens. Bioelectron. 19(5), 411-416 (2003)

3. Wachter, E.A., Thundat, T.: Micromechanical sensors for chemical and physical measurements. Rev. Sci. Instrum. 66, 3662 (1995)

4. Zhang, W., Baskaran, R., Turner, K.L.: Effect of cubic nonlinearity on auto-parametrically amplified resonant MEMS mass sensor. Sens. Actuators A, Phys. 102(1-2), 139-150 (2002)

5. Khater, M., Abdel-Rahman, E., Nayfeh, A.: A mass sensing technique for electrostatically-actuated mems. In: International Design Engineering Technical Conferences and Computers and Information in Engineering Conference, ASME 2009, San Diego, California, USA (2009)

6. Spletzer, M., Raman, A., Sumali, H., Sullivan, J.P.: Highly sensitive mass detection and identification using vibration localization in coupled microcantilever arrays. Appl. Phys. Lett. 92, 114102 (2008)

7. Yin, S.H., Epureanu, B.I.: Experimental enhanced nonlinear dynamics and identification of attractor morphing modes for damage detection. J. Vib. Acoust. 129, 763 (2007)

8. Strogatz, S.H.: Nonlinear Dynamics and Chaos: With Applications to Physics, Biology, Chemistry, and Engineering. Perseus Books, New York (2001)

9. Nayfeh, A.H., Balachandran, B.: Applied Nonlinear Dynamics. Wiley, New York (1995)

10. Epureanu, B.I., Yin, S.H., Dowell, E.H.: Enhanced nonlinear dynamics for accurate identification of stiffness loss in a thermo-shielding panel. Nonlinear Dyn. 39(1), 197-211 (2005)

11. Ghafari, S.H., Golnaraghi, F., Ismail, F.: Effect of localized faults on chaotic vibration of rolling element bearings. Nonlinear Dyn. 53(4), 287-301 (2008)

12. Wu, Y.T., Shyu, K.K., Chen, T.R., Guo, W.Y.: Using threedimensional fractal dimension to analyze the complexity of fetal cortical surface from magnetic resonance images. Nonlinear Dyn., 1-8 (2009)

13. Bienstman, J., Vandewalle, J., Puers, R.: The autonomous impact resonator: a new operating principle for a silicon resonant strain gauge. Sens. Actuators A, Phys. 66(1-3), 40-49 (1998)

14. Wang, Y.C., Adams, S.G., Thorp, J.S., MacDonald, N.C., Hartwell, P., Bertsch, F.: Chaos in MEMS, parameter estimation and its potential application. IEEE Trans. Circuits Syst. I, Fundam. Theory Appl. 45(10), 1013-1020 (1998) 
15. DeMartini, B.E., Butterfield, H.E., Moehlis, J., Turner, K.L.: Chaos for a microelectromechanical oscillator governed by the nonlinear Mathieu equation. J. Microelectromech. Syst. 16(6), 1314-1323 (2007)

16. De, S.K., Aluru, N.R.: Complex oscillations and chaos in electrostatic microelectromechanical systems under superharmonic excitations. Phys. Rev. Lett. 94(20), 204101 (2005)

17. Najar, F., Nayfeh, A.H., Abdel-Rahman, E.M., Choura, S., El-Borgi, S.: Dynamics and global stability of beam-based electrostatic microactuators. J. Vib. Control, 1-4 (2010)

18. Liu, S., Davidson, A., Lin, Q.: Simulation studies on nonlinear dynamics and chaos in a MEMS cantilever control system. J. Micromech. Microeng. 14(7), 1064-1073 (2004)

19. Lu, M.S.C., Fedder, G.K.: Position control of parallel-plate microactuators for probe-based data storage. J. Microelectromech. Syst. 13(5), 759-769 (2004)

20. Towfighian, S., Heppler, G.R., Abdel-Rahman, E.M.: Analysis of a chaotic electrostatic micro-oscillator. J. Comput. Nonlinear Dyn. 6, 011001 (2011)
21. Towfighian, S., Seleim, A., Abdel-Rahman, E.M., Heppler, G.R.: A large-stroke electrostatic micro-actuator. J. Micromech. Microeng. 21, 075023 (2011)

22. Senturia, S.D.: Microsystem Design. Kluwer Academic, Norwell (2001)

23. Najar, F., Nayfeh, A.H., Abdel-Rahman, E.M., Choura, S., El-Borgi, S.: Nonlinear analysis of MEMS electrostatic microactuators: primary and secondary resonances of the first mode. J. Vib. Control 16(9), 1321-1349 (2010)

24. Simoyi, R.H., Wolf, A., Swinney, H.L.: One-dimensional dynamics in a multicomponent chemical reaction. Phys. Rev. Lett. 49(4), 245-248 (1982)

25. Metropolis, N., Stein, M.L., Stein, P.R.: Finite limit sets for transformations on the unit interval. J. Comb. Theory 15, 25-43 (1973)

26. Carnahan, B., Luther, H.A., Wilkes, J.O.: Applied Numerical Methods. Wiley, New York (1969) 